Pure and Applied Mathematics Quarterly

Volume 1, Number 3

(Special Issue: In Memory of

Armand Borel, Part 2 of 3 )

$543-589,2005$

\title{
Maximal Representations of Surface Groups: Symplectic Anosov Structures
}

\author{
Marc Burger, Alessandra Iozzi \\ François Labourie, Anna Wienhard \\ Dedicated to the memory of Armand Borel \\ with affection and admiration
}

\begin{abstract}
Let $G$ be a connected semisimple Lie group such that the associated symmetric space $\mathcal{X}$ is Hermitian and let $\Gamma_{g}$ be the fundamental group of a compact orientable surface of genus $g \geq 2$. We survey the study of maximal representations of $\Gamma_{g}$ into $G$, that is the subset of $\operatorname{Hom}\left(\Gamma_{g}, G\right)$ characterized by the maximality of the Toledo invariant ([17] and [15]). Then we concentrate on the particular case $G=\operatorname{Sp}(2 n, \mathbb{R})$, and we show that if $\rho$ is any maximal representation then the image $\rho\left(\Gamma_{g}\right)$ is a discrete, faithful realizations of $\Gamma_{g}$ as a Kleinian group of complex motions in $\mathcal{X}$ with an associated Anosov system, and whose limit set in an appropriate compactification of $\mathcal{X}$ is a rectifiable circle.
\end{abstract}

\section{Contents}

1. Introduction 544

2. Hermitian Symmetric Spaces and Examples 547

3. The Toledo Invariant and Maximal Representations 552

4. Tube Type Subdomains and Maximal Representations 559

5. Tight Homomorphisms 562

6. Symplectic Anosov Structures 569

7. Bounded Cohomology at Use 571

Received May 24, 2005.

A.I. and A.W. were partially supported by FNS grant PP002-102765. 


\section{INTRODUCTION}

Let $\Gamma_{g}$ be the fundamental group of a compact orientable topological surface $\Sigma_{g}$ of genus $g \geq 2$. For a general real algebraic group $G$ the representation variety $\operatorname{Hom}\left(\Gamma_{g}, G\right)$ is a natural geometric object which reflects properties both of the discrete group $\Gamma_{g}$ and of the algebraic group $G$ and enjoys an extremely rich structure. For example, $\operatorname{Hom}\left(\Gamma_{g}, G\right)$ is not only a topological space, but also a real algebraic variety, which in addition parametrizes flat principal $G$-bundles over $\Sigma_{g}$; furthermore, it admits an action of the group of automorphisms of $\Gamma_{g}$ by precomposition which commutes with the action by postcomposition with (inner) automorphisms of $G$. It is natural to consider homomorphisms up to conjugation, thus we introduce the topological quotient

$$
\operatorname{Rep}\left(\Gamma_{g}, G\right):=\operatorname{Hom}\left(\Gamma_{g}, G\right) / G
$$

although this quotient is not necessarily a Hausdorff space, it contains a large part which is Hausdorff, namely the space $\operatorname{Rep}_{r e d}\left(\Gamma_{g}, G\right)$ of homomorphisms with reductive image modded out by $G$-conjugation. The general theme of this note is the study of certain connected components of $\operatorname{Hom}\left(\Gamma_{g}, G\right)$ or $\operatorname{Rep}\left(\Gamma_{g}, G\right)$ analogous to Teichmüller space, and their relation to geometric and dynamical structures on $\Sigma_{g}$.

Recall that if $G=\mathrm{PU}(1,1)$, the space $\operatorname{Rep}\left(\Gamma_{g}, G\right)$ has $4 g-3$ connected components ([34], [36]), two of which are homeomorphic to $\mathbb{R}^{6 g-6}$ and correspond to the two Teichmüller spaces $\mathcal{T}_{g}$ - one for each orientation of $\Sigma_{g}$ - that is to the space of marked complex, alternatively hyperbolic, structures on the topological surface $\Sigma_{g}$.

If on the other hand $G=\mathrm{SL}(3, \mathbb{R})$, Goldman and Choi proved [21] that one of the three connected components of $\operatorname{Hom}\left(\Gamma_{g}, G\right)[42]$ parametrizes convex projective structures on $\Sigma_{g}$, that is diffeomorphisms of $\Sigma_{g}$ with $\Omega / \Gamma$, where $\Gamma<\operatorname{SL}(3, \mathbb{R})$ is a faithful discrete image of $\Gamma_{g}$ and $\Omega \subset \mathbb{P}\left(\mathbb{R}^{3}\right)$ is a convex invariant domain; incidentally, this component coincides with the Hitchin component that we define below.

If $G=\operatorname{PSL}(2, \mathbb{C})$, there is an open subset of $\operatorname{Rep}\left(\Gamma_{g}, G\right)$ consisting of all quasiFuchsian deformations of $\Gamma_{g}$, which is diffeomorphic to the product $\mathcal{T}_{g} \times \mathcal{T}_{g}$ of two copies of Teichmüller space. 
In each of these three cases, a representation $\rho$ belonging to such a "special component" in $\operatorname{Rep}\left(\Gamma_{g}, G\right)$ is faithful and with discrete image, and $\rho\left(\Gamma_{g}\right)<G$ gives rise, as a Kleinian group, to many interesting dynamical and geometric structures.

When $G$ is a simple split real Lie group - such as $G=\operatorname{PSL}(n, \mathbb{R}), \operatorname{PSp}(2 n, \mathbb{R})$, $\mathrm{PO}(n, n)$ or $\mathrm{PO}(n, n+1)$ - Hitchin singled out a component $\operatorname{Rep}_{H}\left(\Gamma_{g}, G\right)$ of $\operatorname{Rep}\left(\Gamma_{g}, G\right)$ which he proved, using Higgs bundle techniques, to be diffeomorphic to $\mathbb{R}^{\left|\chi\left(\Sigma_{g}\right)\right| \operatorname{dim} G}$, [42] and which is now commonly known as Hitchin component. For example, if $G=\operatorname{PSL}(n, \mathbb{R})$ for $n \geq 2$, this component is the one containing the homomorphisms of $\Gamma_{g}$ into $\mathrm{SL}(n, \mathbb{R})$ obtained by composing a hyperbolization with the $n$-dimensional irreducible representation of $\mathrm{SL}(2, \mathbb{R})$. As Hitchin however points out [42], the analytic point of view does not shed any light on the geometric significance of the representations in this component.

Recently the concept of Anosov representation, which links the surface $\Sigma_{g}$ to flag manifolds associated to $G$ was introduced in [50] and used to show that, if $G=\operatorname{PSL}(n, \mathbb{R})$, representations in the Hitchin component are discrete and faithful, and that they provide quasiisometric embeddings of $\Gamma_{g}$ into $G$, [50], [51].

In parallel, Goncharov and Fock developed for surfaces with nonempty boundary and in the case of split real Lie groups a tropical-algebro-geometric viewpoint of $\operatorname{Rep}\left(\Gamma_{g}, G\right)$, singling out positive real points in $\operatorname{Rep}\left(\Gamma_{g}, G\right)$ which correspond to discrete and faithful representations, [30], [29].

There is another natural extension of the case $G=\mathrm{PU}(1,1)$ in a different direction, that is to connected semisimple Lie groups $G$ such that the associated symmetric space $\mathcal{X}$ admits a $G$-invariant complex structure, just like in the case of the Poincaré disk. This includes notably groups like $\mathrm{SU}(p, q), \mathrm{Sp}(2 n, \mathbb{R}), \mathrm{SO}^{*}(2 n)$, $\mathrm{SO}(2, n)$. Symmetric spaces with this property are called Hermitian.

In the same framework, the topology and the number of connected components of the space of reductive representations into $\mathrm{SU}(p, q)$ and $\operatorname{Sp}(2 n, \mathbb{R})$ have been studied in a series of papers by Bradlow, García-Prada, Gothen, Mundet i Riera and Xia ([6], [4], [7], [37], [32], [68]), extending the analytic approach introduced by Hitchin.

The additional feature for symmetric spaces which are Hermitian is the presence of a Kähler form $\omega_{\mathcal{X}}$ on $\mathcal{X}$ which allows to associate to every representation $\rho: \Gamma_{g} \rightarrow G$ a characteristic number, called the Toledo invariant $\mathrm{T}_{\rho}$ (see $\S 3$ ), which is constant on connected components of $\operatorname{Hom}\left(\Gamma_{g}, G\right)$ and which satisfies a Milnor-Wood type inequality

$$
\left|\mathrm{T}_{\rho}\right| \leq\left|\chi\left(\Sigma_{g}\right)\right| \operatorname{rk} \mathcal{X}
$$


where $\mathrm{rk}_{\mathcal{X}}$ is the real rank of $X$. A representation is maximal if equality holds in (1.1), and the set $\operatorname{Hom}_{\max }\left(\Gamma_{g}, G\right)$ of such representations is then a union of components of $\operatorname{Hom}\left(\Gamma_{g}, G\right)$. If $G=\operatorname{PU}(1,1)$, Goldman proved in [34] that maximal representations are exactly those lying in the two Teichmüller components.

In the first part of this article we illustrate, mostly without proofs, results concerning the geometric significance of maximal representations. To fix the notation, let $G:=\mathbf{G}(\mathbb{R})^{\circ}$, where $\mathbf{G}$ is a semisimple real algebraic group and assume that the symmetric space $\mathcal{X}$ associated to $G$ is Hermitian. In complete analogy with Goldman's theorem, any maximal representation $\rho: \Gamma_{g} \rightarrow G$ is injective with discrete image (Theorem 4.6). This fact depends on a careful study of the Zariski closure $\mathbf{L}$ of the image $\rho\left(\Gamma_{g}\right)$ and the fact that there is an essential restriction on $L:=\mathbf{L}(\mathbb{R})^{\circ}$, namely that it is reductive and it preserves a subHermitian symmetric space of $\mathcal{X}$ which is of tube type and maximal with respect to this property. On the constructive side, the study of maximal representations into $G$ does not reduce to the study of classical Teichmüller space; in fact, if $\mathcal{X}$ is of tube type, any representation which is the composition of a hyperbolization $\Gamma_{g} \rightarrow \mathrm{SU}(1,1)$ with the homomorphism $\mathrm{SU}(1,1) \rightarrow G$ associated to the realization of the Poincaré disk diagonally in a maximal polydisk in $\mathcal{X}$ can be deformed into a representation with Zariski dense image in $\mathbf{G}$ (Theorem 4.7); such a representation is by construction maximal. For examples of discrete representations into $\mathrm{SU}(1, n)$ with prescribed Toledo invariant see [35].

These results are proven in greater generality in [15], where for the representation of the fundamental group of a surface with boundary, we define a Toledo invariant whose definition and properties however require some vigorous use of bounded cohomology. In the context of this paper, continuous bounded cohomology will appear as a tool in the proofs; in particular it allows to define the notion of tight homomorphism, more general and flexible than that of maximal representation, and which is an essential tool to study the geometric properties of the inclusion $\mathcal{X}_{L} \hookrightarrow \mathcal{X}$, where $\mathcal{X}_{L}$ is the subsymmetric space associated to $L$ (see above). A systematic study of tight homomorphisms and the companion notion of tight embedding is the subject matter of [16] and a few highlights of this theory are presented in $\S 5$.

While the first part of the paper is expository, in the second part we give an elementary treatment of a certain number of results on maximal representations into the symplectic group $\operatorname{Sp}(V)$ of a real symplectic vector space $V$. The results are stated in $\S 6$ and their proofs in $\S 8$ are independent of the rest of the paper (see $\S 8)$.

Observe at this point that $\operatorname{Sp}(V)$ is at the same time real split, and hence falls into the context of the Hitchin component, and is the group of automorphisms of the Siegel upper half space, a fundamental class of Hermitian symmetric spaces. 
We have the inclusion

$$
\operatorname{Rep}_{H}\left(\Gamma_{g}, \operatorname{Sp}(V)\right) \subset \operatorname{Rep}_{\text {max }}\left(\Gamma_{g}, \operatorname{Sp}(V)\right),
$$

but while the representations in the Hitchin component are all irreducible [50], there are (at least when $\operatorname{dim} V \geq 4$ ) components of maximal representations which contain reducible representations, so that the above inclusion is strict.

For a representation $\rho: \Gamma_{g} \rightarrow \operatorname{Sp}(V)$ and a fixed hyperbolization $\Sigma$ of $\Sigma_{g}$, we associate the flat symplectic bundle $E^{\rho}$ over the unit tangent bundle $T^{1} \Sigma$ of $\Sigma$ with fiber $V$. The geodesic flow lifts canonically to a flow $g_{t}^{\rho}$ on $E^{\rho}$; we adapt some of the ideas in [50] to our situation and, combining them with the results in $\S 5$ and $\S 7$, prove that if $\rho$ is maximal then $E^{\rho}$ is the sum of two continuous Lagrangian subbundles $E_{+}^{\rho} \oplus E_{-}^{\rho}$ on which $g_{t}^{\rho}$ acts contracting and expanding respectively. Moreover, this bundle will also come with a field of complex structures in each fiber, exchanging $E_{ \pm}^{\rho}$ and positive for the symplectic structure (see $\S 5$ ). As a consequence, one deduces that any maximal representation $\rho: \Gamma_{g} \rightarrow \operatorname{Sp}(V)$ is a quasiisometric embedding, where $\operatorname{Sp}(V)$ is equipped with a standard invariant metric. This implies that the action of the mapping class group $\operatorname{Out}\left(\Gamma_{g}\right)$ on $\operatorname{Rep}_{\text {max }}\left(\Gamma_{g}, \operatorname{Sp}(V)\right)$ is properly discontinuous.

Acknowledgments: The authors thank Domingo Toledo for bringing to their attention [45] and [62] and Domingo Toledo and Nicolas Monod for useful comments on a preliminary version of the paper.

\section{Hermitian Symmetric Spaces and Examples}

Let $\mathcal{X}$ be a symmetric space and let $G:=\operatorname{Isom}(\mathcal{X})^{\circ}$ be the connected component of its group of isometries. Recall that $\mathcal{X}$ is Hermitian if it admits a $G$-invariant complex structure. An equivalent definition is that $\mathcal{X}$ is a Hermitian manifold such that every point $x \in \mathcal{X}$ is the isolated fixed point of an isometric involution $s_{x}$. In this paper we shall consider only symmetric spaces of noncompact type.

Let $J$ be the $G$-invariant complex structure and let $g: T \mathcal{X} \times{ }_{p} T \mathcal{X} \rightarrow \mathbb{R}$ be the Riemannian metric, where $T \mathcal{X} \times_{p} T \mathcal{X}$ denotes the fibered product over the projection $p: T \mathcal{X} \rightarrow \mathcal{X}$. Then

$$
\omega_{\mathcal{X}}(X, Y):=g(J X, Y)
$$

defines a $G$-invariant differential two-form on $\mathcal{X}$ which is nondegenerate.

Lemma 2.1. Let $\mathcal{X}$ be a symmetric space and $G=\operatorname{Isom}(\mathcal{X})^{\circ}$. Then any $G$ invariant differential form on $\mathcal{X}$ is closed. 
Proof. Let $\alpha$ be a $G$-invariant differential $k$-form on $\mathcal{X}$ and let $s \in \operatorname{Isom}(\mathcal{X})$ be the geodesic symmetry at a basepoint $0 \in \mathcal{X}$. Since $G$ is normal in $\operatorname{Isom}(\mathcal{X})$, then $s g s \in G$ and hence $s \alpha$ is also $G$-invariant, since

$$
g(s \alpha)=s^{2} g(s \alpha)=s(s g s) \alpha=s \alpha .
$$

Moreover, since $\left.s\right|_{T_{0} \mathcal{X}}=-I d$ we have that $(s \alpha)_{0}=(-1)^{k} \alpha_{0}$, and since $\alpha$ and $s \alpha$ are both $G$-invariant, the equality $(s \alpha)_{x}=(-1)^{k} \alpha_{x}$ holds for every $x \in \mathcal{X}$. Since $d \alpha$ is also $G$-invariant, from

$$
(-1)^{k} d \alpha=d(s \alpha)=s(d \alpha)=(-1)^{k+1} d \alpha
$$

we deduce that $d \alpha=0$.

The immediate consequence of the above lemma is that a Hermitian symmetric space $\mathcal{X}$ is a Kähler manifold with Kähler form $\omega_{\mathcal{X}}$. Furthermore, using the existence of a Kähler form on $\mathcal{X}$, one can prove that for an irreducible symmetric space $\mathcal{X}$ being Hermitian is equivalent to the center of a maximal compact subgroup of $\operatorname{Isom}(\mathcal{X})^{\circ}$ having positive dimension (and in fact being one-dimensional).

A fundamental result which makes the study of Hermitian symmetric spaces accessible to techniques from function theory à la Bergmann is the following theorem of Harish-Chandra which for classical domains is due to E. Cartan, [20].

Theorem 2.2 (Harish-Chandra, [40]). Any Hermitian symmetric space of noncompact type is biholomorphic to a bounded domain in a complex vector space.

The bounded realization $\mathcal{D} \subset \mathbb{C}^{N}$ of a Hermitian symmetric space $\mathcal{X}$ has a natural compactification, namely the topological closure $\overline{\mathcal{D}}$ in $\mathbb{C}^{N}$, on which $G:=\operatorname{Isom}(\mathcal{X})^{\circ}$ acts by restriction of birational isomorphism of $\mathbb{C}^{N}$. The Shilov boundary $\check{S}$ is a subset of the topological boundary $\partial \mathcal{D}$ of the bounded domain; it can be defined in function theoretical terms, and it is also the unique closed $G$-orbit in $\overline{\mathcal{D}}$. It is a homogeneous space of the form $G / Q$, where $Q$ is a (specific) maximal parabolic subgroup of $G$, and plays a prominent role in our study, for example as target of appropriate boundary maps. Notice that only if $\mathcal{X}$ is of real rank one, the Shilov boundary coincides with the whole boundary $\partial \mathcal{D}$.

Recall that the rank $\mathrm{rk}_{\mathcal{X}}$ of a symmetric space $\mathcal{X}$ is the maximal dimension of a flat subspace, that is an isometric copy of Euclidean space.

Expositions of different aspects of the geometry of Hermitian symmetric spaces are [46], [23], [58], [57], and [67].

2.1. Examples of Hermitian Symmetric Spaces. We give here examples of two families of Hermitian symmetric spaces which are of fundamental nature and with which we shall illustrate our results. 
2.1.1. $\mathrm{SU}(W)$. Let $W$ be a complex vector space of dimension $n$, and $h(\cdot, \cdot)$ a nondegenerate Hermitian form of signature $(p, q), p \leq q$, so that $p$ is the maximal dimension of a subspace $L \subset W$ on which the restriction $\left.h(\cdot, \cdot)\right|_{L}$ is positive definite. A model for the symmetric space associated to

$$
\mathrm{SU}(W):=\{g \in \mathrm{SL}(W): h(g x, g y)=h(x, y), \forall x, y \in W\}
$$

is

$$
\mathcal{X}_{\mathrm{SU}(W)}:=\left\{L \in \operatorname{Gr}_{p}(W):\left.h(\cdot, \cdot)\right|_{L} \text { is positive definite }\right\}
$$

which, as an open subset of the Grassmannian $\operatorname{Gr}_{p}(W)$ of $p$-dimensional subspaces of $W$, is a complex manifold on which $G$ acts by automorphisms. To realize $\mathcal{X}_{\mathrm{SU}(W)}$ as a bounded domain, fix $W_{+} \in \mathcal{X}_{\mathrm{SU}(W)}$, and let $W_{-}:=W_{+}^{\perp}$ be its orthogonal complement with respect to the form $h$. Since the orthogonal projection $\operatorname{pr}_{\epsilon}: W \rightarrow W_{\epsilon}, \epsilon \in\{+,-\}$, is an isomorphism for $\epsilon=+$ when restricted to any $L \in \mathcal{X}_{\mathrm{SU}(W)}$, we can define

$$
E: \mathcal{X}_{\mathrm{SU}(W)} \rightarrow \operatorname{Lin}\left(W_{+}, W_{-}\right)
$$

by

$$
E(L):=\mathrm{pr}_{-} \circ\left(\left.\mathrm{pr}_{+}\right|_{L}\right)^{-1} .
$$

It is easy to see that this defines a biholomorphic map from $\mathcal{X}_{\mathrm{SU}(W)}$ to the bounded domain

$$
\mathcal{D}_{\mathrm{SU}(W)}:=\left\{A \in \operatorname{Lin}\left(W_{+}, W_{-}\right): I d-A^{*} A \text { is positive definite }\right\}
$$

where the adjoint is taken with respect to the structures of the unitary spaces $\left(W_{+},\left.h(\cdot, \cdot)\right|_{W_{+}}\right)$and $\left(W_{-},-\left.h(\cdot, \cdot)\right|_{W_{-}}\right)$. Moreover the closure $\overline{\mathcal{X}}_{\mathrm{SU}(W)}$ of $\mathcal{X}_{\mathrm{SU}(W)}$ in $\operatorname{Gr}_{p}(W)$ is mapped by $E$ to

$$
\overline{\mathcal{D}_{\mathrm{SU}(W)}}=\left\{A \in \operatorname{Lin}\left(W_{+}, W_{-}\right): I d-A^{*} A \text { is positive semidefinite }\right\} .
$$

To determine the preimage of the Shilov boundary in the hyperboloid model $\mathcal{X}_{\mathrm{SU}(W)}$, observe that there are precisely $(p+1)$ orbits of $\mathrm{SU}(W)$ in $\overline{\mathcal{X}}_{\mathrm{SU}(W)}$, only one of which is closed, namely the Grassmannian of maximal isotropic subspaces

$$
\operatorname{Is}_{p}(W):=\left\{L \in \operatorname{Gr}_{p}(W):\left.h(\cdot, \cdot)\right|_{L}=0\right\},
$$

which is hence sent via $E$ to the Shilov boundary

$$
\check{S}_{\mathrm{SU}(W)}=\left\{A \in \operatorname{Lin}\left(W_{+}, W_{-}\right): I d-A^{*} A=0\right\} \subset \overline{\mathcal{D}_{\mathrm{SU}(W)}}
$$

of the bounded domain $\mathcal{D}_{\mathrm{SU}(W)}$. The real rank of $\mathcal{X}_{\mathrm{SU}(W)}$ is $p$.

Identifying $W$ with $\mathbb{C}^{p+q}$ in such a way that $h$ is the standard Hermitian form of signature $(p, q)$, we denote $\mathrm{SU}(p, q):=\mathrm{SU}(W)$ and

$$
\mathcal{D}_{p, q}=\left\{Z \in M_{q, p}(\mathbb{C}): I d-{ }^{t} \bar{Z} Z \text { is positive definite }\right\}
$$

the corresponding bounded domain with Shilov boundary

$$
\check{S}_{p, q}=\left\{Z \in M_{q, p}(\mathbb{C}): I d-{ }^{t} \bar{Z} Z=0\right\} \subset \overline{\mathcal{D}_{p, q}} .
$$


In particular $\mathcal{D}_{1,1}$ is the Poincaré disk.

2.1.2. The Symplectic Group. Let $V$ be a real vector space equipped with a symplectic form $\langle\cdot, \cdot\rangle$, that is a nondegenerate antisymmetric bilinear form. In particular $V$ must be even dimensional and we fix $\operatorname{dim} V=2 n$. The group

$$
\operatorname{Sp}(V):=\{g \in \mathrm{GL}(V):\langle g x, g y\rangle=\langle x, y\rangle, \forall x, y \in V\}
$$

is the real symplectic group. The fact that on a complex vector space the imaginary part of a nondegenerate Hermitian form is a symplectic form for the underlying real structure suggests to introduce the space

$$
\begin{aligned}
\mathcal{X}:=\{J \in \mathrm{GL}(V): & J \text { is a complex structure on } V \text { and } \\
& h_{J}(x, y):=\langle x, J y\rangle+i\langle x, y\rangle \text { is a positive definite } \\
& \text { Hermitian form on }(V, J)\},
\end{aligned}
$$

so that, if $J \in \mathcal{X}$, then $\Re h_{J}(x, y)=\langle x, J y\rangle$ is a symmetric positive definite form. It is easy to see that, among complex structures on $V$, this property characterizes the elements of $\mathcal{X}$. Furthermore, for the transitive action by conjugation of $\operatorname{Sp}(V)$ on $\mathcal{X}$, the stabilizer of $J$ is a maximal compact subgroup isomorphic to $\mathrm{U}(n)$ and hence $\mathcal{X}$ is the symmetric space $\mathcal{X}_{\mathrm{Sp}(V)}$ associated to $\operatorname{Sp}(V)$; in particular, since the center of $\mathrm{U}(n)$ has positive dimension, $\mathcal{X}_{\mathrm{Sp}(V)}$ is Hermitian symmetric and, as such, there is a $\operatorname{Sp}(V)$-invariant complex structure on $\mathcal{X}_{\mathrm{Sp}(V)}$ which one can explicit as follows.

Let $V_{\mathbb{C}}$ be the complexification of $V$ and let $\sigma: V_{\mathbb{C}} \rightarrow V_{\mathbb{C}}$ be the complex conjugation $\sigma(x+i y):=x-i y$ for $x, y \in V$. Then there is a bijective correspondence between complex structures $J$ on $V$ and decompositions $V_{\mathbb{C}}=L_{+} \oplus L_{-}$into complex subspaces satisfying $\sigma\left(L_{ \pm}\right)=L_{\mp}$, given by the eigenspace decomposition of $J \otimes_{\mathbb{C}} \mathbb{1}$ into $\pm i$-eigenspaces. If now $\langle\cdot, \cdot\rangle_{\mathbb{C}}$ is the complexification of $\langle\cdot, \cdot\rangle$, then

$$
h(x, y):=i\langle x, \sigma(y)\rangle_{\mathbb{C}}
$$

is a nondegenerate Hermitian form of signature $(n, n)$ on $V_{\mathbb{C}}$; if in particular $J \in \mathcal{X}_{\mathrm{Sp}(V)}$, then the restriction $\left.h\right|_{L_{+} \times L_{+}}$is positive definite, so that we obtain a map

$$
\begin{aligned}
\mathcal{X}_{\mathrm{Sp}(V)} & \rightarrow \mathcal{X}_{\mathrm{SU}\left(V_{\mathbb{C}}\right)} \\
J & \mapsto L_{+}
\end{aligned}
$$

which is equivariant with respect to the natural homomorphism

$$
\begin{aligned}
\lambda: \mathrm{Sp}(V) & \rightarrow \mathrm{SU}\left(V_{\mathbb{C}}\right) \\
g & \mapsto g \otimes_{\mathbb{C}} \mathbb{1} .
\end{aligned}
$$

Since $\mathcal{X}_{\mathrm{SU}\left(V_{\mathbb{C}}\right)}$ inherits a natural complex structure as an open subset of the Grassmannian $\mathrm{Gr}_{n}\left(V_{\mathbb{C}}\right)$ of $n$-planes in $V_{\mathbb{C}}$ and

$$
M:=\left\{L \in \operatorname{Gr}_{n}\left(V_{\mathbb{C}}\right):\left.\langle\cdot, \cdot\rangle_{\mathbb{C}}\right|_{L \times L} \equiv 0\right\}
$$


is an algebraic subvariety of $\mathrm{Gr}_{n}\left(V_{\mathbb{C}}\right)$, then $\mathcal{X}_{\mathrm{SU}\left(V_{\mathbb{C}}\right)} \cap M$ acquires a natural complex structure as an open subset of $M$. But

$$
\left.\langle\cdot, \cdot\rangle_{\mathbb{C}}\right|_{L_{ \pm} \times L_{ \pm}} \equiv 0
$$

for all $J \in \mathcal{X}_{\mathrm{Sp}(V)}$, so that the map in (2.3) is a $\lambda$-equivariant bijection between $\mathcal{X}_{\mathrm{Sp}(V)}$ and $\mathcal{X}_{\mathrm{SU}\left(V_{\mathbb{C}}\right)} \cap M$ by the use of which the complex structure on $\mathcal{X}_{\mathrm{SU}\left(V_{\mathbb{C}}\right)} \cap M$ defines the $\operatorname{Sp}(V)$-invariant complex structure on $\mathcal{X}_{\mathrm{Sp}(V)}$.

Let us denote by $\mathcal{S}_{\mathrm{Sp}(V)}:=\mathcal{X}_{\mathrm{SU}\left(V_{\mathbb{C}}\right)} \cap M$ the Siegel space associated to $\operatorname{Sp}(V)$ with its $\operatorname{Sp}(V)$-action via the homomorphism $\lambda$.

To determine the bounded domain realization of $\mathcal{X}_{\mathrm{Sp}(V)}$, it is enough to observe that - with the notations of $\S 2.1 .1$, where $W=V_{\mathbb{C}}$ - the Siegel space $\mathcal{S}_{\mathrm{Sp}(V)}$ is mapped by the map $E$ defined in (2.1) to the subdomain

$$
\mathcal{D}_{\mathrm{Sp}(V)}:=\left\{A \in \mathcal{D}_{\mathrm{SU}\left(V_{\mathbb{C}}\right)}:\left.\langle\cdot, A \cdot\rangle_{\mathbb{C}}\right|_{W_{+} \times W_{+}} \text {is symmetric }\right\},
$$

and, accordingly, $\overline{\mathcal{S}}_{\mathrm{Sp}(V)}$ is mapped to

$$
\overline{\mathcal{D}_{\mathrm{Sp}(V)}}:=\left\{A \in \overline{\mathcal{D}_{\mathrm{SU}\left(V_{\mathbb{C}}\right)}}:\left.\langle\cdot, A \cdot\rangle_{\mathbb{C}}\right|_{W_{+} \times W_{+}} \text {is symmetric }\right\} .
$$

One can verify again that the closure $\overline{\mathcal{S}_{\mathrm{Sp}(V)}}$ in $\operatorname{Gr}_{n}\left(V_{\mathbb{C}}\right)$ decomposes into $(n+1)$ orbits under the symplectic group, only one of which is closed, namely

$$
\operatorname{Is}_{n}\left(V_{\mathbb{C}}\right) \cap \overline{\mathcal{S}_{\operatorname{Sp}(V)}}=\left\{L \in \operatorname{Gr}_{n}\left(V_{\mathbb{C}}\right):\left.\langle\cdot, \cdot\rangle_{\mathbb{C}}\right|_{L \times L}=0 \text { and }\left.h\right|_{L \times L} \equiv 0\right\}
$$

and hence is the preimage, under the map $E$ in (2.1), of the Shilov boundary in the bounded domain realization of $\mathcal{S}_{\mathrm{Sp}(V)}$. To give an intrinsic description of the Shilov boundary observe that, since we have the alternative description

$$
\operatorname{Is}_{n}\left(V_{\mathbb{C}}\right) \cap \overline{\mathcal{S}_{\operatorname{Sp}(V)}}=\left\{L \in \operatorname{Gr}_{n}\left(V_{\mathbb{C}}\right):\left.\langle\cdot, \cdot\rangle_{\mathbb{C}}\right|_{L \times L}=0 \text { and } \sigma(L)=L\right\},
$$

we conclude that the map

$$
\begin{aligned}
\mathcal{L}(V) & \rightarrow \mathrm{Is}_{n}\left(V_{\mathbb{C}}\right) \cap \overline{\mathcal{S}_{\mathrm{Sp}(V)}} \\
L & \mapsto L \otimes \mathbb{C},
\end{aligned}
$$

where $\mathcal{L}(V)$ is the space of Lagrangians in $V$, is an $\operatorname{Sp}(V)$-equivariant bijection. Thus the space of Lagrangian subspaces is, as a $\operatorname{Sp}(V)$-homogeneous space, isomorphic to the Shilov boundary of the bounded domain $\mathcal{D}_{\mathrm{Sp}(V)}$.

If we identify $V$ with the direct sum of $n$ symplectic planes, that is copies of $\mathbb{R}^{2}$ with the standard symplectic form, then accordingly we denote the symplectic group by $\operatorname{Sp}(2 n, \mathbb{R})$ and the Siegel space by $\mathcal{S}_{n}$. 


\section{The Toledo Invariant and Maximal Representations}

Let $\Sigma_{g}$ be a compact oriented surface of genus $g \geq 2, G$ a connected semisimple Lie group with finite center and associated symmetric space $\mathcal{X}$ which we assume to be Hermitian, and $\rho: \Gamma_{g} \rightarrow G$ a homomorphism. Then there is a smooth $\Gamma_{g}$-equivariant map $\tilde{f}: \widetilde{\Sigma_{g}} \rightarrow \mathcal{X}$, where $\widetilde{\Sigma_{g}}$ denotes the universal covering of $\Sigma_{g}$, which can be obtained by lifting a smooth section of the flat bundle $\Gamma_{g} \backslash\left(\widetilde{\Sigma_{g}} \times\right.$ $\mathcal{X}) \rightarrow \Sigma_{g}$ with contractible fiber $\mathcal{X}$. The pullback $\tilde{f}^{*} \omega_{\mathcal{X}}$ is then a $\Gamma$-invariant differential form on $\widetilde{\Sigma_{g}}$, which hence descends to a form on $\Sigma_{g}$. Since any two such sections are homotopic and hence the map $\tilde{f}$ is unique up to $\rho$-equivariant homotopy, the result of the integration over $\Sigma_{g}$ of any two forms obtained in this way does not depend on the particular choice of a section: we can hence define the Toledo invariant of $\rho$

$$
\mathrm{T}_{\rho}:=\frac{1}{2 \pi} \int_{\Sigma_{g}} \tilde{f}^{*}\left(\omega_{\mathcal{X}}\right)
$$

Normalizing the metric on $\mathcal{X}$ once and for all so that the minimal holomorphic sectional curvature is -1 , we can summarize the properties of the Toledo invariant in the following

Proposition 3.1. There exists a rational number $\ell_{\mathcal{X}} \in \mathbb{Q}$ such that the Toledo invariant $\mathrm{T}_{\rho}$ of a representation $\rho: \Gamma_{g} \rightarrow G$ has the following properties:

(1) $\mathrm{T}_{\rho} \in \ell \mathcal{X} \mathbb{Z}$

(2) the map $\mathrm{T}: \operatorname{Hom}\left(\Gamma_{g}, G\right) \rightarrow \ell_{\mathcal{X}} \mathbb{Z}$ is constant on connected components of the representation variety, and

(3) $\left|\mathrm{T}_{\rho}\right| \leq\left|\chi\left(\Sigma_{g}\right)\right| \mathrm{rk}_{\mathcal{X}}$, where $\mathrm{rk}_{\mathcal{X}}$ is the real rank of $\mathcal{X}$.

REMARK 3.2. (1) The constant $\ell_{\mathcal{X}}$ can be explicitly computed from the restricted root system of the real Lie group $G$ (see [14]). In fact, the metric of minimal holomorphic sectional curvature -1 on $\mathcal{X}$ is the $\ell_{\mathcal{X}}$-multiple of the Bergmann metric given by the bounded domain realization of $\mathcal{X}$.

(2) The inequality in Proposition 3.1(3) is due to J. Milnor in the case $G=$ $\mathrm{SL}(2, \mathbb{R})[54]$, to V. Turaev in the case $G=\operatorname{Sp}(2 n, \mathbb{R})[64]$, to A. Domic and D. Toledo in the classical cases [24] and to J. L. Clerc and B. Ørsted in the general case [22].

We want to give now a very concrete interpretation of the Toledo invariant of a representation $\Gamma_{g} \rightarrow G$ in the case in which $G=\operatorname{Isom}(\mathcal{X})^{\circ}$, in terms of generators of $\Gamma_{g}$; this is very much in the spirit of Milnor's formula for the Euler number (see [54]). 
Let $0 \in \mathcal{X}$ be a basepoint and $K$ its stabilizer in $G$. We already alluded to the fact that the center of $K$ is of positive dimension (see $\S 2$ ). In fact, the $\mathbb{C}$ vector space structure on the tangent space $T_{0} \mathcal{X}$ gives an action of $\mathrm{U}(1):=\{z \in$ $\mathbb{C}:|z|=1\}$ which can be "integrated", in the sense that there is a continuous homomorphism

$$
u_{0}: \mathrm{U}(1) \rightarrow K
$$

such that for $z \in \mathrm{U}(1)$ the differential of the isometry of $\mathcal{X}$ defined by $u_{0}(z)$ at 0 is the multiplication $v \mapsto z v$ for all $v \in T_{0} \mathcal{X}$. In particular, since $K$ acts on $T_{0} \mathcal{X}$ faithfully by $\mathbb{C}$-linear maps, the image of $u_{0}$ is in the center $Z(K)$ of $K$ and in fact coincides with $Z(K)$ when $\mathcal{X}$ is irreducible.

Assume hence for the following discussion that $\mathcal{X}$ is irreducible. The homomorphism in (3.1) induces a homomorphism on the level of fundamental groups $\mathbb{Z} \rightarrow \pi_{1}(K)=\pi_{1}(G)$ and hence an isomorphism

$$
\mathbb{Z} \rightarrow \pi_{1}(G) / \pi_{1}(G)_{t o r} .
$$

Denoting by $\widehat{G}$ the covering of $G$ associated to $\pi_{1}(G)_{t o r}$, we obtain a topological central extension

$$
0 \longrightarrow \mathbb{Z} \longrightarrow \widehat{G} \longrightarrow G \longrightarrow(e) \text {. }
$$

The commutator map $\widehat{G} \times \widehat{G} \rightarrow \widehat{G}$ factors via $\mathbb{Z}$ to give rise to a smooth map

$$
\begin{aligned}
G \times G & \rightarrow \widehat{G} \\
(a, b) & \mapsto \widehat{[a, b]} .
\end{aligned}
$$

Given a standard presentation of $\Gamma_{g}$,

$$
\Gamma_{g}=\left\langle a_{1}, b_{1}, \ldots, a_{g}, b_{g}: \prod_{i=1}^{g}\left[a_{i}, b_{i}\right]=e\right\rangle,
$$

to any homomorphism $\rho: \Gamma_{g} \rightarrow G$ we can thus associate

$$
\prod_{i=1}^{g}\left[\rho\left(\widehat{\left.a_{i}\right), \rho}\left(b_{i}\right)\right] \in \mathbb{Z}\right.
$$

and the same argument as in [54], shows that

$$
\mathrm{T}_{\rho}=\ell_{\mathcal{X}} \prod_{i=1}^{g}\left[\rho\left(\widehat{\left.a_{i}\right), \rho}\left(b_{i}\right)\right] .\right.
$$

As an immediate consequence we have that the Toledo invariant depends continuously on the representation and hence (1) and (2) of Proposition 3.1 follow at once. The proof of part (3) of the same proposition is more delicate; one efficient way to prove it uses the value of the simplicial area of $\Sigma_{g}$ and the value of the 
norm of the bounded Kähler class in bounded cohomology determined by Domic and Toledo [24] and by Clerc and Ørsted [22], and will be explained in $\S 5$.

If $G=\mathrm{PU}(1,1)$ and $\mathcal{X}$ is the Poincaré disk, the constant $\ell_{\mathcal{X}}$ in Proposition 3.1 equals 1 and hence for the Toledo invariant of a homomorphism

$$
\rho: \Gamma_{g} \rightarrow \mathrm{PU}(1,1)
$$

we have that $\mathrm{T}_{\rho} \in \mathbb{Z}$ and $\left|\mathrm{T}_{\rho}\right| \leq 2 g-2$. Thus $\mathrm{T}_{\rho}$ can achieve at most $4 g-3$ values. In fact:

Theorem 3.3 (Goldman, [34], [36]). The fibers of

$$
\begin{aligned}
\operatorname{Hom}\left(\Gamma_{g}, \mathrm{PU}(1,1)\right) & \rightarrow\{-(2 g-2), \ldots, 0, \ldots, 2 g-2\} \\
\rho & \mapsto \quad \mathrm{T}_{\rho}
\end{aligned}
$$

are exactly the connected components of $\operatorname{Hom}\left(\Gamma_{g}, \mathrm{PU}(1,1)\right)$. Moreover $\mathrm{T}_{\rho}=$ $\pm(2 g-2)$ if and only if $\rho$ is a hyperbolization, that is it is faithful with discrete image.

Let now $G$ be any connected semisimple Lie group with finite center such that the associated symmetric space $\mathcal{X}$ is Hermitian. Inspired by Proposition 3.1(3) and by Goldman's result, we give the following

Definition 3.4. We say that a representation $\rho: \Gamma_{g} \rightarrow G$ is maximal if $\mathrm{T}_{\rho}=$ $\pm \chi\left(\Sigma_{g}\right) \mathrm{rk} \mathcal{X}$

In the sequel we shall show that, in this degree of generality, maximal representations contain a remarkable amount of structure. Historically, the following result of $\mathrm{H}$. Kneser seems to be the birth certificate of this theme.

Theorem 3.5 (Kneser, [45]). Let $f: \Sigma_{g} \rightarrow \Sigma_{h}$ be a continuous map of compact orientable surfaces, where $h \geq 2$. If $d_{f}$ is the degree of $f$ we have

$$
\left|d_{f}(h-1)\right| \leq|g-1|,
$$

with equality if and only if $f$ is homotopic to a covering map, necessarily of degree $d_{f}$.

G. Lusztig observed that this inequality is a consequence of Milnor's inequality (see [26] for this remark as well as a proof using harmonic maps), by taking a hyperbolization

$$
\rho: \pi_{1}\left(\Sigma_{h}\right) \rightarrow \mathrm{PU}(1,1)
$$

of $\Sigma_{h}$ and considering the Toledo invariant of the composed homomorphism

$$
\rho \circ f_{*}: \pi_{1}\left(\Sigma_{g}\right) \rightarrow \mathrm{PU}(1,1) .
$$

Actually, continuing along these lines and applying Goldman's theorem characterizing maximal representations into $\mathrm{PU}(1,1)$ leads to a proof of the equality case in Kneser's theorem. The reader might find instructive to fill in the details. 
3.1. Examples of Maximal Representations. It is a nontrivial and remarkable geometric fact that any maximal flat in a Hermitian symmetric space can be "complexified" hence leading to the existence of maximal polydisks.

Definition 3.6. A maximal polydisk in $\mathcal{X}$ is a subHermitian symmetric space in $\mathcal{X}$ isomorphic to the $r$-fold Cartesian product $\left(\mathcal{D}_{1,1}\right)^{r}$ of the Poincaré disk, where $r=\mathrm{rk}_{\mathcal{X}}$.

The fact that maximal polydisks exist (and are all conjugate under $\operatorname{Isom}(\mathcal{X})^{\circ}$ ) $[67$, p. 280] is a crucial ingredient in some of the examples to follow and their generalization (see Theorem 4.7).

EXAMPLE 3.7. The embedding

$$
\begin{aligned}
& \left(\mathcal{D}_{1,1}\right)^{p} \quad \rightarrow \quad \mathcal{D}_{p, q} \\
& \left(z_{1}, \ldots, z_{p}\right) \mapsto\left(\begin{array}{ccc}
z_{1} & & \\
& \ddots & \\
& & z_{p} \\
0 & \ldots & 0 \\
\vdots & & \vdots \\
0 & \ldots & 0
\end{array}\right)
\end{aligned}
$$

is isometric and holomorphic and hence defines a maximal polydisk $\mathcal{P} \subset \mathcal{D}_{p, q}$ associated to the obvious homomorphism

$$
\tau_{\mathcal{P}}: \mathrm{SU}(1,1)^{p} \rightarrow \mathrm{SU}(p, q) .
$$

EXAMPLE 3.8. Let $\mathbb{R}^{2 n}=\mathbb{R}^{2} \oplus \cdots \oplus \mathbb{R}^{2}$ be the direct sum of $n$ symplectic planes. Then the embedding

$$
\begin{aligned}
& \left(\mathcal{X}_{\mathrm{Sp}(2, \mathbb{R})}\right)^{n} \rightarrow \quad \mathcal{X}_{\mathrm{Sp}(2 n, \mathbb{R})} \\
& \left(J_{1}, \ldots, J_{n}\right) \mapsto\left(\begin{array}{ccc}
J_{1} & & \\
& \ddots & \\
& & J_{n}
\end{array}\right)
\end{aligned}
$$

defines a maximal polydisk $\mathcal{P} \subset \mathcal{X}_{\mathrm{Sp}(2 n, \mathbb{R})}$ associated to the obvious homomorphism

$$
\tau_{\mathcal{P}}:(\operatorname{Sp}(2, \mathbb{R}))^{n} \rightarrow \operatorname{Sp}(2 n, \mathbb{R})
$$

Now we present some examples of maximal representations, though the proof of their maximality is not necessarily immediate at this point.

Example 3.9. Let $\mathcal{X}$ be any Hermitian symmetric space of rank $r$, and $\mathcal{P} \subset \mathcal{X}$ a maximal polydisk with associated homomorphism

$$
\tau_{\mathcal{P}}: \operatorname{SU}(1,1)^{r} \rightarrow G:=\operatorname{Isom}(\mathcal{X})^{\circ} .
$$


Given now $r$ orientation preserving hyperbolizations $h_{1}, \ldots, h_{r}: \Gamma_{g} \rightarrow \mathrm{SU}(1,1)$, the representation

$$
\begin{aligned}
h: \Gamma_{g} & \longrightarrow \mathrm{SU}(1,1)^{r} \\
\gamma & \mapsto\left(h_{1}(\gamma), \ldots, h_{r}(\gamma)\right)
\end{aligned}
$$

as well as the composition

$$
\tau_{\mathcal{P}} \circ h: \Gamma_{g} \rightarrow G
$$

are maximal.

ExAmple 3.10. Let $h: \Gamma_{g} \rightarrow \mathrm{SL}(2, \mathbb{R})$ be an orientation preserving hyperbolization and let $\rho_{2 n}: \mathrm{SL}(2, \mathbb{R}) \rightarrow \mathrm{SL}(2 n, \mathbb{R})$ be the $2 n$-dimensional irreducible representation of $\operatorname{SL}(2, \mathbb{R})$. Since $\rho_{2 n}(\mathrm{SL}(2, \mathbb{R}))$ preserves the standard symplectic form on $\mathbb{R}^{2 n}$, we obtain a representation

$$
\rho_{2 n} \circ h: \Gamma_{g} \rightarrow \operatorname{Sp}(2 n, \mathbb{R})
$$

which can be proven to be maximal. Observe that such a representation $\rho_{2 n} \circ h$ is in the Hitchin component $\operatorname{Rep}_{H}\left(\Gamma_{g}, \operatorname{Sp}(2 n, \mathbb{R})\right)$ (see $\left.\S 1\right)$.

The fact that $\tau_{\mathcal{P}} \circ h$ and $\rho_{2 n} \circ h$ are maximal depends on the property of $\tau_{\mathcal{P}}$ and $\rho_{2 n}$ being "tight homomorphisms", a concept to which we shall return in $\S 5$.

ExAmple 3.11. Because of Proposition 3.1(2), any deformation of any of the above representations is maximal. In particular, all representations in the Hitchin component are maximal.

EXAMPLE 3.12. Let $\Sigma_{g}$ be a compact oriented surface of genus $g \geq 2$. Our objective is to give an example of maximal representation with Zariski dense image in $\operatorname{Sp}(4, \mathbb{R})$ constructed via an explicit deformation of the representation in Example 3.9. This was triggered by a comment of Toledo, who pointed out that a disk diagonally embedded into a polydisk does not determine uniquely the polydisk.

To this end, write $\Sigma_{g}$ as the sum of two surfaces $\Sigma_{A}, \Sigma_{B}$ identified along a separating simple closed loop $\gamma$ on which we choose a basepoint $p$ and realize $\Gamma_{g}$ as an amalgamated product $\Gamma_{A} *\langle\gamma\rangle \Gamma_{B}$ of $\Gamma_{A}:=\pi_{1}\left(\Sigma_{A}, p\right)$ and $\Gamma_{B}:=\pi_{1}\left(\Sigma_{B}, p\right)$ over the infinite cyclic subgroup $\langle\gamma\rangle$.

Let $h: \Gamma_{g} \rightarrow \operatorname{PSp}(2, \mathbb{R})^{2}$ be defined by $h(\gamma):=\left(h_{1}(\gamma), h_{2}(\gamma)\right)$, where $h_{1}, h_{2}$ : $\Gamma_{g} \rightarrow \operatorname{PSp}(2, \mathbb{R})$ are two inequivalent hyperbolizations, and let us choose some lift to $\operatorname{Sp}(2, \mathbb{R})^{2}$, denoted again by $h$ with a small abuse of notation,

$$
\begin{aligned}
h: \Gamma_{g} & \rightarrow \operatorname{Sp}(2, \mathbb{R})^{2} \\
\gamma & \mapsto\left(h_{1}(\gamma), h_{2}(\gamma)\right) .
\end{aligned}
$$




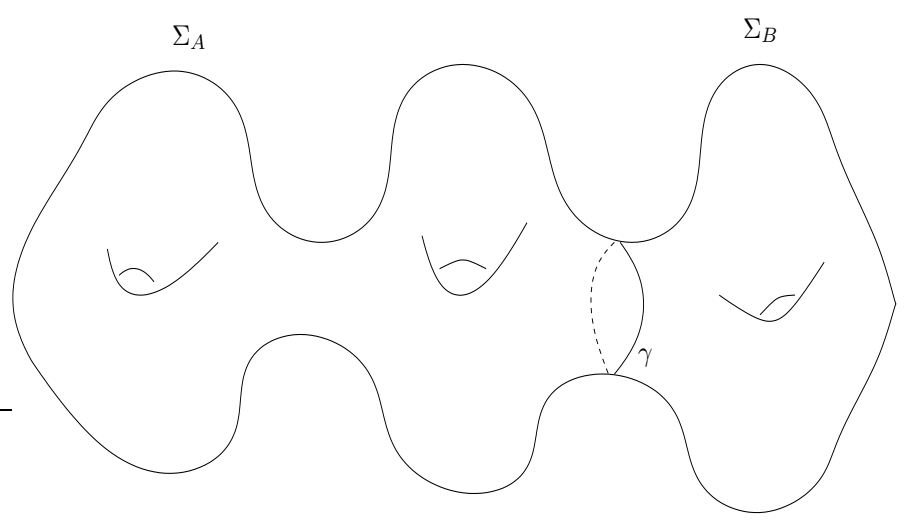

Figure 1.

We shall assume that:

(*) $h(\gamma) \in \Delta$, where $\Delta$ is the diagonal of $\operatorname{Sp}(2, \mathbb{R})^{2}$, and

$(* *)$ the restriction of the two hyperbolic structures to $\Sigma_{A}$ and to $\Sigma_{B}$ are inequivalent,

and we denote by

$$
\begin{aligned}
h_{A}: \Gamma_{A} & \rightarrow \operatorname{Sp}(2, \mathbb{R})^{2} \\
\alpha & \mapsto\left(h_{1, A}(\alpha), h_{2, A}(\alpha)\right)
\end{aligned}
$$

and

$$
\begin{aligned}
h_{B}: \Gamma_{B} & \rightarrow \operatorname{Sp}(2, \mathbb{R})^{2} \\
\beta & \mapsto\left(h_{1, B}(\beta), h_{2, B}(\beta)\right)
\end{aligned}
$$

the restrictions of $h$ to $\Gamma_{A}$ and $\Gamma_{B}$ respectively.

Let now $\mathbb{R}^{4}=\mathbb{R}^{2} \oplus \mathbb{R}^{2}$ be the sum of two standard symplectic real planes (as in 2.1.2), so that

$$
\mathrm{Sp}(4, \mathbb{R})=\left\{g=\left(\begin{array}{ll}
A & B \\
C & D
\end{array}\right) \in \mathrm{GL}(4, \mathbb{R}):{ }^{t} g\left(\begin{array}{ll}
J & 0 \\
0 & J
\end{array}\right) g=\left(\begin{array}{ll}
J & 0 \\
0 & J
\end{array}\right)\right\}
$$

where $J=\left(\begin{array}{cc}0 & 1 \\ -1 & 0\end{array}\right)$. Consider the homomorphism

$$
\begin{aligned}
\tau_{\mathcal{P}}: \operatorname{Sp}(2, \mathbb{R})^{2} & \rightarrow \operatorname{Sp}(4, \mathbb{R}) \\
(A, D) & \mapsto\left(\begin{array}{cc}
A & 0 \\
0 & D
\end{array}\right)
\end{aligned}
$$


associated to the maximal polydisk

$$
\mathcal{P}=\left\{\left(\begin{array}{cc}
J_{1} & 0 \\
0 & J_{2}
\end{array}\right) \in \mathcal{X}_{\mathrm{Sp}(4, \mathbb{R})}: J_{1}, J_{2} \text { are complex structures on } \mathbb{R}^{2}\right\} .
$$

Then the centralizer $\mathcal{Z}$ in $\operatorname{Sp}(4, \mathbb{R})$ of the image $\tau_{\mathcal{P}}(\Delta)$ of the diagonal is

$$
\mathcal{Z}=\left\{\left(\begin{array}{ll}
a I d_{2} & b I d_{2} \\
c I d_{2} & d I d_{2}
\end{array}\right):\left(\begin{array}{ll}
a & b \\
c & d
\end{array}\right) \in \mathrm{O}(2)\right\}
$$

Denoting by $\operatorname{Int}(z)$ the conjugation by $z \in \mathcal{Z}$, the homomorphisms $\tau_{\mathcal{P}} \circ h_{A}$ and $\operatorname{Int}(z) \circ \tau_{\mathcal{P}} \circ h_{B}$ coincide on $\langle\gamma\rangle($ see $(*))$. Thus by the universal property of amalgams, there is a unique homomorphism

$$
\rho_{z}: \Gamma_{g} \rightarrow \operatorname{Sp}(4, \mathbb{R})
$$

whose restriction to $\Gamma_{A}$ is $\tau_{\mathcal{P}} \circ h_{A}$ and to $\Gamma_{B}$ is $\operatorname{Int}(z) \circ \tau_{\mathcal{P}} \circ h_{B}$.

Proposition 3.13. With the above notations:

(1) For every $z \in \mathcal{Z}^{\circ}$ the representation $\rho_{z}: \Gamma_{g} \rightarrow \operatorname{Sp}(4, \mathbb{R})$ is maximal, and

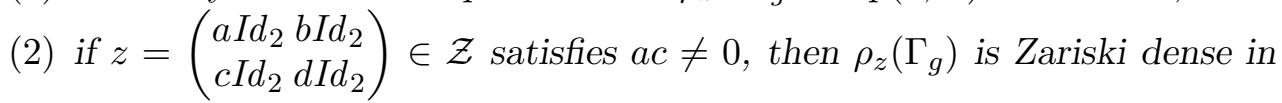
$\operatorname{Sp}(4, \mathbb{R})$.

Proof. (1) If $z=I d, \rho_{I d}$ is the composition of $h: \Gamma_{g} \rightarrow \operatorname{Sp}(2, \mathbb{R})^{2}$ with $\tau_{\mathcal{P}}$ : $\mathrm{Sp}(2, \mathbb{R})^{2} \rightarrow \mathrm{Sp}(4, \mathbb{R})$. The latter homomorphism is associated to an embedding realizing $\left(\mathcal{X}_{\mathrm{Sp}(2, \mathbb{R})}\right)^{2}$ as a maximal polydisk in the Siegel space $\mathcal{X}_{\mathrm{Sp}(4, \mathbb{R})}$ and hence $\rho_{I d}$ is maximal (see Example 3.9). Every $z \in \mathcal{Z}^{\circ}$ can be connected to $I d$ by a continuous path, thus $\rho_{z}$ is in the same component of $\operatorname{Hom}\left(\Gamma_{g}, \operatorname{Sp}(4, \mathbb{R})\right)$ as $\rho_{I d}$ and thus maximal.

(2) It follows from $(* *)$ that the image $\rho_{z}\left(\Gamma_{g}\right)$ is Zariski dense in the algebraic group $L<\operatorname{Sp}(4, \mathbb{R})$ generated by $P:=\tau_{\mathcal{P}}\left(\operatorname{Sp}(2, \mathbb{R})^{2}\right)$ and $z P z^{-1}$. Now the condition on $z$ guarantees that the Lie algebra $\mathfrak{p}$ of $P$ is strictly contained in the Lie algebra $\mathfrak{l}$ of $L$. But it is easily verified that the representation of $\operatorname{Sp}(2, \mathbb{R})^{2}$ on the Lie algebra $\mathfrak{s p}(4, \mathbb{R})$ of $\operatorname{Sp}(4, \mathbb{R})$ obtained by composing $\tau_{\mathcal{P}}$ with the adjoint representation is a sum of $\mathfrak{p}$ and the irreducible representation in dimension 4 , tensor product of the standard 2-dimensional representation of $\operatorname{Sp}(2, \mathbb{R})$ with itself. This implies that $\mathfrak{l}=\mathfrak{s p}(4, \mathbb{R})$ and hence proves the proposition.

EXAMPLE 3.14. A smooth fiber bundle over a surface $\Sigma_{g}$ with typical fiber $\Sigma_{n}$ leads, via the monodromy representation on the first homology group of the fiber, to a representation

$$
\rho: \Gamma_{g} \rightarrow \operatorname{Sp}(2 n, \mathbb{Z}) .
$$

For such representations, D. Kotschick [47] showed that

$$
\left|\mathrm{T}_{\rho}\right| \leq \frac{1}{2}\left|\chi\left(\Sigma_{g}\right)\right|(n-1),
$$


and in particular $\rho$ is far from being maximal. On the other hand D. Toledo has given examples of maximal representations into $\operatorname{Sp}(4 n, \mathbb{Z})$ for all $n \geq 1$, [62].

\section{Tube Type Subdomains and Maximal Representations}

Let $G$ be a semisimple real algebraic group with associated symmetric space $\mathcal{X}$ of Hermitian type. In view of Goldman's theorem (see Theorem 3.3), a basic question concerning a maximal representation $\rho: \Gamma_{g} \rightarrow G$ is whether it is faithful with discrete image. In addition, when $\mathcal{X}$ is not the Poincaré disk, $\rho\left(\Gamma_{g}\right)$ cannot be a lattice in $G$ and thus in this general setting there is the question of determining how "large" the image of $\rho$ can be. Concerning the latter question, it is natural to turn one's attention to the Zariski closure $\mathbf{L}$ of $\rho\left(\Gamma_{g}\right)$. While in the preceding examples we have seen that $\mathbf{L}(\mathbb{R})$ can be a product $\operatorname{Sp}(2, \mathbb{R})^{r}$, or more interestingly $\operatorname{Sp}(4, \mathbb{R})$, it turns out however that there are restrictions on $\mathbf{L}$ and that moreover the determination of these restrictions is an essential step in order to answer the question about faithfulness and discreteness of $\rho$.

To this purpose, an instructive special case is the family of Hermitian symmetric spaces of real rank one, that is the complex hyperbolic spaces $\mathcal{D}_{1, q}$. This case, beyond $q=1$, was examined by Toledo and in order to state his result we recall that a complex geodesic is an isomorphic copy of $\mathcal{D}_{1,1}$ in $\mathcal{D}_{1, q}$; equivalently, complex geodesics are obtained by taking the exponential of a complex line in $T_{x} \mathcal{D}_{1, q}$, for $x \in \mathcal{D}_{1, q}$; they constitute the maximal polydisks in $\mathcal{D}_{1, q}$.

Theorem 4.1 (Toledo, [63]). Any maximal representation $\rho: \Gamma_{g} \rightarrow \mathrm{PU}(1, q)$ stabilizes a complex geodesic.

Since the stabilizer in $\mathrm{PU}(1, q)$ of a complex geodesic is, modulo a compact kernel, isomorphic to $\mathrm{PU}(1,1)$, Goldman's theorem [34] applies, and thus $\rho$ is basically obtained via a hyperbolization of $\Sigma_{g}$.

The proof of Toledo's theorem is very much in the spirit of the GromovThurston proof of Mostow rigidity theorem and uses notably $\ell^{1}$-homology and smearing. (Incidentally, $\ell^{1}$-homology will play a role also in our treatment of the Milnor-Wood type inequality in Proposition 3.1(3) as described in $\S 5$ ). A special case of Theorem 4.1 was already proven by Toledo in [61] using harmonic mappings techniques. In the same spirit, taking up Hitchin's approach via Higgs bundles, Bradlow, García-Prada and Gothen made a comprehensive study of the topology of the connected components of $\operatorname{Hom}_{r e d}\left(\Gamma_{g}, \mathrm{PU}(p, q)\right)$ and obtained in particular for the maximal representations the following 
Theorem 4.2 (Bradlow-García-Prada-Gothen, [4]). Assume that $1 \leq p \leq q$. If $\rho: \Gamma_{g} \rightarrow \mathrm{PU}(p, q)$ is maximal and reductive, then its image is contained in $\mathrm{P}(\mathrm{U}(p, p) \times \mathrm{U}(q-p))$ up to conjugation.

This result had been previously obtained by L. Hernández in the case $p=2$, [41]. Moreover, an analogous result has been proven by Bradlow, García-Prada and Gothen for $\mathrm{SO}^{*}(2 n)$, if $n$ is odd [5].

An equivalent way of stating the theorem asserts that $\rho\left(\Gamma_{g}\right)$ preserves a Hermitian symmetric subspace of $\mathcal{D}_{p, q}$ conjugate to $\mathcal{D}_{p, p}$. In order to understand the situation in general, the relevant concept here is the one of tube type domain. For instance, the Hermitian symmetric space associated to PU $(1,1)$ has a realization as upper half plane but, unlike the bounded domain realization, this type of realization is not available for all Hermitian symmetric spaces.

Definition 4.3. A Hermitian symmetric space $\mathcal{X}$ is of tube type if it is biholomorphic to a domain

$$
\{u+i v: u \in V, v \in \Omega\} \subset V \oplus i V
$$

where $V$ is a real vector space and $\Omega \subset V$ is a proper open cone.

EXAmple 4.4. The space $\mathcal{D}_{p, q}$ associated to $\mathrm{SU}(p, q)$ is of tube type if and only if $p=q$, in which case it is biholomorphic to

$$
\operatorname{Herm}_{p}(\mathbb{C})+i \operatorname{Herm}_{p}^{+}(\mathbb{C}),
$$

where $\operatorname{Herm}_{p}(\mathbb{C})$ is the real vector space of Hermitian matrices and $\operatorname{Herm}_{p}^{+}(\mathbb{C})$ is the open cone of positive definite ones. The biholomorphism is given explicitly by restricting to $\mathcal{D}_{p, p}$ the Cayley transformation

$$
\begin{aligned}
\mathcal{C}: M_{p, p}(\mathbb{C}) & \rightarrow M_{p, p}(\mathbb{C}) \\
Z & \mapsto \frac{i(I d+Z)}{(I d-Z)} .
\end{aligned}
$$

The Cayley transformation $\mathcal{C}$ sends the real Zariski open subset of the Shilov boundary $\check{S}_{p, p}$ of $\mathcal{D}_{p, p}$ consisting of matrices $Z \in M_{p, p}(\mathbb{C})$ such that $\operatorname{det}(I-Z) \neq 0$ bijectively into $\operatorname{Herm}_{p}(\mathbb{C})$. Considering $\operatorname{Herm}_{p}^{+}(\mathbb{C})$ as an open cone in the tangent space of $\operatorname{Herm}_{p}(\mathbb{C})$ at 0 , and taking its image under the differential at 0 of $\mathcal{C}^{-1}$, one obtains a cone $\Omega$ in the tangent space $T_{-I d} \check{S}_{p, p}$ which is invariant under the stabilizer of $-I d$ in $\mathrm{SU}(p, p)$. Translating this cone by the $\mathrm{SU}(p, p)$-action, one obtains a smooth family of open cones $\Omega_{x} \subset T_{x} \check{S}_{p, p}$, that is a causal structure on the Shilov boundary, which is $\mathrm{SU}(p, p)$-invariant.

EXAmPle 4.5. Under the same Cayley transformation, the bounded domain realization of the Siegel space is sent biholomorphically to

$$
\operatorname{Sym}_{n}(\mathbb{R})+i \operatorname{Sym}_{n}^{+}(\mathbb{R}),
$$


where $\operatorname{Sym}_{n}(\mathbb{R})$ denotes the vector space consisting of real symmetric $n \times n$ matrices, and realizes the symmetric space associated to $\operatorname{Sp}(2 n, \mathbb{R})$ as a tube type domain.

The open cone $\mathrm{Sym}^{+}(\mathbb{R})$ defines in the same way as above an $\operatorname{Sp}(V)$-invariant causal structure on $\mathcal{L}(V)$ which will be used in the proof of Corollary 6.3.

The examples above serve to illustrate the general fact that the Shilov boundary of a bounded symmetric domain of tube type admits an invariant causal structure. Among bounded symmetric domains, this property characterizes those of tube type. The general classification of Hermitian symmetric spaces relative to the notion of tube type is as follows:

\begin{tabular}{c|c}
\hline Tube Type & Non-Tube Type \\
\hline $\mathrm{SU}(p, p)$ & $\mathrm{SU}(p, q)$ \\
& $p<q$ \\
\hline $\mathrm{Sp}(2 n, \mathbb{R})$ & \\
\hline $\mathrm{SO}^{*}(2 n)$ & $\mathrm{SO}^{*}(2 n)$ \\
$n$ even & $n$ odd \\
\hline $\mathrm{SO}(2, n)$ & \\
\hline$E_{7}(-25)$ & $E_{6}(-14)$ \\
\hline
\end{tabular}

where $E_{7}(-25)$ and $E_{6}(-14)$ correspond to the exceptional Hermitian symmetric spaces of complex dimension 27 and 16 respectively.

An essential feature of a Hermitian symmetric space of rank $\mathrm{rk}_{\mathcal{X}}$ is that (holomorphically embedded) maximal Hermitian subdomains of tube type always exist, are of rank $\mathrm{rk}_{\mathcal{X}}$ and are all conjugate.

Notice that in the rank one case, that is for complex hyperbolic $n$-space, the notion of maximal tube type subdomain and maximal polydisk coincide. This ambiguity left open the correct generalization of Toledo's theorem until the construction of a maximal representation with Zariski dense image in a tube type domain [17], of which Example 3.12 is a particular case.

We can finally state the structure theorem for maximal representations.

TheOREM 4.6 ([17], [15]). Let $\mathbf{G}$ be a connected semisimple real algebraic group and assume that the symmetric space $\mathcal{X}_{G}$ associated to $G:=\mathbf{G}(\mathbb{R})^{\circ}$ is Hermitian symmetric. Let $\rho: \Gamma_{g} \rightarrow G$ be a maximal representation. Then:

(1) $\Gamma_{g}$ acts properly discontinuously, via $\rho$, on $\mathcal{X}_{G}$;

(2) the Zariski closure $\mathbf{L}$ of $\rho\left(\Gamma_{g}\right)$ is reductive;

(3) the connected component $L:=\mathbf{L}(\mathbb{R})^{\circ}$ stabilizes a maximal tube type subdomain $T \subset \mathcal{X}_{G}$; 
(4) the symmetric space $\mathcal{X}_{L}$ associated to $L$ is Hermitian of tube type and the isometric embedding $\mathcal{X}_{L} \hookrightarrow T$ is tight.

We illustrate the above theorem in the examples of $\S 3$. For the notion of tight embedding see $\S 5$.

EXAMPLE. 3.9 The orientation preserving hyperbolizations $h_{1}, \ldots, h_{r}$ fall into $s$ equivalence classes modulo $\mathrm{SU}(1,1)$-equivalence, with $1 \leq s \leq r$; the group $L$ is then isomorphic to $\mathrm{SU}(1,1)^{s}$ and $\mathcal{X}_{L}$ is a product of $s$-subdiagonals in the maximal polydisk $\mathcal{P}$. Any diagonal disk in $\mathcal{P}$ determines the same maximal tube type domain $T$ and $\mathcal{X}_{L} \subset \mathcal{P} \subset T$. The first statement of the theorem is obvious in this example.

EXAMPLE. 3.10 In this case $L \cong \operatorname{Sp}(2, \mathbb{R}), T=\mathcal{X}_{\mathrm{Sp}(2 n, \mathbb{R})}$, and $\mathcal{X}_{L} \subset \mathcal{X}_{\mathrm{Sp}(2 n, \mathbb{R})}$ is a geodesically embedded disk, holomorphic if and only if $n=1$. Item (1) of the theorem is obvious also in this case.

Example. 3.12 Under the conditions of Proposition 3.13(2), the maximal representation

$$
\rho_{z}: \Gamma_{g} \rightarrow \operatorname{Sp}(4, \mathbb{R})
$$

has Zariski dense image, so $L=\operatorname{Sp}(4, \mathbb{R})$ and $\mathcal{X}_{L}=T=\mathcal{X}_{\mathrm{Sp}(4, \mathbb{R})}$ by construction. On the other hand, Theorem 4.6(1) implies that $\rho_{z}$ is injective with discrete image, a fact that in this case is not at all obvious from the construction.

As alluded to earlier, Example 3.12 is a particular case of a general fact which we now state. Let $r=\operatorname{rk} \mathcal{X}$, let $\mathcal{P} \subset \mathcal{X}$ be a maximal polydisk and

$$
\tau_{\mathcal{P}}: \operatorname{SU}(1,1)^{r} \rightarrow \operatorname{Isom}(\mathcal{X})^{\circ} .
$$

the associated homomorphism.

Theorem 4.7 ([17], [15]). Assume that $\mathcal{X}$ is of tube type and let $\rho_{0}: \Gamma_{g} \rightarrow$ $\operatorname{Isom}(\mathcal{X})^{\circ}$ be the maximal representation obtained by composing a hyperbolization of $\Gamma_{g} \rightarrow \mathrm{SU}(1,1)$ with the diagonal embedding of $\mathrm{SU}(1,1)$ in $\mathrm{SU}(1,1)^{r}$ followed by $\tau_{\mathcal{P}}$. Then $\rho_{0}$ admits a continuous deformation $\rho_{t}: \Gamma_{g} \rightarrow \operatorname{Isom}(\mathcal{X})^{\circ}$, for $t \geq 0$, such that $\rho_{t}\left(\Gamma_{g}\right)$ is Zariski dense in $\operatorname{Isom}(\mathcal{X})^{\circ}$ for $t>0$.

Observe that $\rho_{t}$, being a continuous deformation of a maximal representation, is maximal as well.

\section{Tight Homomorphisms}

A fundamental role in the study of maximal representations of surface groups is played by tight homomorphisms, which generalize maximal representations of 
surface groups, in that it is a notion defined for any continuous homomorphism of a locally compact group into the group of isometries of a Hermitian symmetric space.

The definition of tight homomorphism rests on basic concepts in bounded continuous cohomology which we briefly recall; for a comprehensive treatment see [56] and [19]. We start with the more familiar concept of continuous group cohomology. For a locally compact group $G$, its continuous cohomology $\mathrm{H}_{\mathrm{c}}^{\bullet}(G, \mathbb{R})$ is the cohomology of the complex $\left(\mathrm{C}\left(G^{\bullet}, \mathbb{R}\right)^{G}, d^{\bullet}\right)$ of $G$-invariant real valued continuous cochains, where $d^{\bullet}$ is the usual homogeneous coboundary. The bounded continuous cohomology $\mathrm{H}_{\mathrm{cb}}^{\bullet}(G, \mathbb{R})$ is then the cohomology of the subcomplex $\left(\mathrm{C}_{\mathrm{b}}\left(G^{\bullet}, \mathbb{R}\right)^{G}, d^{\bullet}\right)$ of $G$-invariant bounded continuous cochains. The complex $\left(\mathrm{C}_{\mathrm{b}}\left(G^{\bullet}, \mathbb{R}\right)^{G}, d^{\bullet}\right)$ equipped with the supremum norm is a complex of Banach spaces with continuous coboundary operators, and hence $\mathrm{H}_{\mathrm{cb}}^{\bullet}(G, \mathbb{R})$ is endowed with a quotient seminorm. Also, the inclusion of the complex of bounded continuous functions into the one of continuous functions gives rise to a comparison map

$$
\mathrm{c}_{G}^{\bullet}: \mathrm{H}_{\mathrm{cb}}^{\bullet}(G, \mathbb{R}) \rightarrow \mathrm{H}_{\mathrm{c}}^{\bullet}(G, \mathbb{R})
$$

which encodes subtle properties of $G$ of geometric and algebraic nature. See [1], [33], [55], [18], [19, § V.13], [13], and also [8], [9], [38], [60], [2], [28], [31], [27], [3], [49], [48] in relation with the existence of quasi-morphisms.

If now $G$ is a connected semisimple Lie group with finite center and associated symmetric space $\mathcal{X}$, we have seen that the complex $\left(\Omega^{\bullet}(\mathcal{X})^{G}, d^{\bullet}\right)$ of $G$-invariant differential forms on $\mathcal{X}$ coincides with its cohomology (see Lemma 2.1) and, in fact, there is a canonical isomorphism [65]

$$
\mathrm{H}_{\mathrm{c}}^{\bullet}(G, \mathbb{R}) \cong \Omega^{\bullet}(\mathcal{X})^{G} .
$$

Let us now specialize to the case of interest to us, namely when $\mathcal{X}$ is Hermitian symmetric and $\omega_{\mathcal{X}} \in \Omega^{2}(\mathcal{X})^{G}$ is its Kähler form. A continuous cocycle defining the class $\kappa_{\mathcal{X}} \in \mathrm{H}_{\mathrm{c}}^{2}(G, \mathbb{R})$ corresponding to $\omega_{\mathcal{X}}$ is

$$
c \mathcal{X}\left(g_{1}, g_{2}, g_{3}\right)=\int_{\Delta\left(g_{1} x, g_{2} x, g_{3} x\right)} \omega_{\mathcal{X}},
$$

where $x \in \mathcal{X}$ is a basepoint and $\Delta\left(g_{1} x, g_{2} x, g_{3} x\right)$ is any smooth two-simplex with geodesic sides and vertices $g_{1} x, g_{2} x, g_{3} x$.

There is a general conjecture of Dupont to the extent that cocycles obtained by integrating $G$-invariant differential forms (of any degree) should be bounded, [25]. In terms of the comparison map, this suggests the following

Question. Let $G$ be a connected semisimple Lie group with finite center. Is the comparison map (5.1) surjective in all degrees? 
This turns out to be true for forms representing specific classes (see [39], [25], [59], [10], [52]) and in particular for the Kähler form was first shown by Dupont [25]. In fact, with the assumed normalization on the metric of $\mathcal{X}$ (see $\S 3$ ), one has the equality

$$
\left\|c_{\mathcal{X}}\right\|_{\infty}=\pi \mathrm{rk}_{\mathcal{X}}
$$

due to Domic and Toledo for classical domains [24] and to Clerc and Ørsted in the general case [22]. Thus $c_{\mathcal{X}}$ defines a continuous bounded class $\kappa_{\mathcal{X}}^{\mathrm{b}} \in \mathrm{H}_{\mathrm{cb}}^{2}(G, \mathbb{R})$ to which we shall refer to the bounded Kähler class; and for which one has the following theorem (see also Proposition 7.4):

Theorem 5.1 (Domic-Toledo [24], Clerc-Ørsted [22]). If the metric on $\mathcal{X}$ is normalized to have minimal holomorphic sectional curvature -1 , then

$$
\left\|\kappa_{\mathcal{X}}^{\mathrm{b}}\right\|=\pi \mathrm{rk} \mathcal{X} .
$$

Given now locally compact groups $H$ and $G$, any continuous homomorphism $\rho: H \rightarrow G$ induces canonical pullbacks $\rho^{\bullet}$ and $\rho_{\mathrm{b}}^{\bullet}$ respectively in continuous and bounded continuous cohomology, by precomposition of continuous (bounded) cochains with $\rho$; the resulting linear maps have the property that the diagram

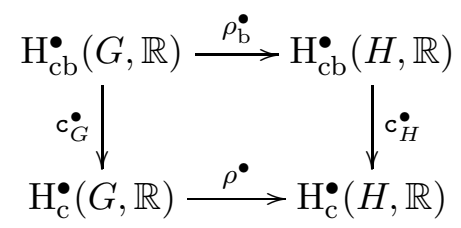

commutes, and moreover the pullback in bounded continuous cohomology is norm decreasing, namely for all $\alpha \in \mathrm{H}_{\mathrm{cb}}^{n}(G, \mathbb{R})$,

$$
\left\|\rho_{\mathrm{b}}^{(n)}(\alpha)\right\| \leq\|\alpha\| \text {. }
$$

Definition 5.2 ([16], [66]). Let $G$ be a connected semisimple group with finite center and such that the associated symmetric space $\mathcal{X}$ is Hermitian, and let $H$ be any locally compact group. A continuous homomorphism $\rho: H \rightarrow G$ is tight if it preserves the norm of the bounded Kähler class, that is if

$$
\left\|\rho_{\mathrm{b}}^{(2)}\left(\kappa_{\mathcal{X}}^{\mathrm{b}}\right)\right\|=\left\|\kappa_{\mathcal{X}}^{\mathrm{b}}\right\|
$$

To motivate this definition, we sketch a proof of the inequality in Proposition 3.1(3). Since $\Sigma_{g}$ is a $K\left(\Gamma_{g}, 1\right)$, we have in particular a canonical isomorphism

$$
\mathrm{H}^{2}\left(\Gamma_{g}, \mathbb{R}\right) \rightarrow \mathrm{H}^{2}\left(\Sigma_{g}, \mathbb{R}\right)
$$

which, if $\kappa_{\mathcal{X}} \in \mathrm{H}_{\mathrm{c}}^{2}(G, \mathbb{R})$ allows us to see $\rho^{(2)}\left(\kappa_{\mathcal{X}}\right) \in \mathrm{H}^{2}\left(\Gamma_{g}, \mathbb{R}\right)$ as a singular class in $\mathrm{H}^{2}\left(\Sigma_{g}, \mathbb{R}\right)$ and evaluate it on the fundamental class $\left[\Sigma_{g}\right] \in \mathrm{H}_{2}\left(\Sigma_{g}, \mathbb{R}\right)$ of $\Sigma_{g}$; recall that $\Sigma_{g}$ is oriented once and for all. Then if

$$
\langle\cdot, \cdot\rangle: \mathrm{H}^{2}\left(\Sigma_{g}, \mathbb{R}\right) \times \mathrm{H}_{2}\left(\Sigma_{g}, \mathbb{R}\right) \rightarrow \mathbb{R}
$$


denotes the pairing, analogously to the classical case of the Euler number, we have

$$
\mathrm{T}_{\rho}=\frac{1}{2 \pi}\left\langle\rho^{(2)}(\kappa \mathcal{X}),\left[\Sigma_{g}\right]\right\rangle .
$$

The proof of the Milnor-Wood type inequality in Proposition 3.1(3) will follow from the interpretation of this invariant in bounded cohomology. To this purpose, following Gromov [39], recall that the $\ell^{1}$-homology of $\Sigma_{g}$ is the homology $\mathrm{H}_{\bullet, \ell^{1}}\left(\Sigma_{g}, \mathbb{R}\right)$ of the complex of singular $\ell^{1}$-chains, while the bounded cohomology $\mathrm{H}_{\mathrm{b}}^{\bullet}\left(\Sigma_{g}, \mathbb{R}\right)$ is the cohomology of the dual Banach space complex; consequently, $\ell^{1}$-homology and bounded cohomology acquire quotient seminorms and there is the canonical pairing

$$
\langle\cdot, \cdot\rangle_{\mathrm{b}}: \mathrm{H}_{\mathrm{b}}^{n}\left(\Sigma_{g}, \mathbb{R}\right) \times \mathrm{H}_{n, \ell^{1}}\left(\Sigma_{g}, \mathbb{R}\right) \rightarrow \mathbb{R}
$$

which satisfies the property that for all $\alpha \in \mathrm{H}_{\mathrm{b}}^{n}\left(\Sigma_{g}, \mathbb{R}\right)$ and all $a \in \mathrm{H}_{n, \ell^{1}}\left(\Sigma_{g}, \mathbb{R}\right)$

$$
\left|\langle\alpha, a\rangle_{\mathrm{b}}\right| \leq\|\alpha\|\|a\|_{\ell^{1}} \text {. }
$$

These notions have been introduced by Gromov for any topological space $X$ and one has the Gromov-Brooks canonical isometric isomorphism (see [39] and [8])

$$
\mathrm{H}_{\mathrm{b}}^{\bullet}\left(\pi_{1}(X), \mathbb{R}\right) \cong \mathrm{H}_{\mathrm{b}}^{\bullet}(X, \mathbb{R}),
$$

a rather deep fact depending on higher homotopy groups being Abelian and hence amenable. In our situation one can explicitly write an isometric isomorphism

$$
\mathrm{H}_{\mathrm{b}}^{2}\left(\Gamma_{g}, \mathbb{R}\right) \cong \mathrm{H}_{\mathrm{b}}^{2}\left(\Sigma_{g}, \mathbb{R}\right)
$$

compatible with the isomorphism in ordinary cohomology, by choosing a hyperbolic metric on $\Sigma_{g}$ and using the technique of straightening simplices.

Starting now with the bounded Kähler class $\kappa_{\mathcal{X}}^{\mathrm{b}} \in \mathrm{H}_{\mathrm{cb}}^{2}(G, \mathbb{R})$, and applying the pullback in bounded cohomology and the isomorphism (5.4), we obtain the class $\rho_{\mathrm{b}}^{(2)}\left(\kappa_{\mathcal{X}}^{\mathrm{b}}\right) \in \mathrm{H}_{\mathrm{b}}^{2}\left(\Sigma_{g}, \mathbb{R}\right)$ which corresponds, using $(5.3)$, to $\rho^{(2)}\left(\kappa_{\mathcal{X}}\right) \in \mathrm{H}^{2}\left(\Sigma_{g}, \mathbb{R}\right)$ under the comparison map in singular cohomology

$$
\mathrm{H}_{\mathrm{b}}^{2}\left(\Sigma_{g}, \mathbb{R}\right) \rightarrow \mathrm{H}^{2}\left(\Sigma_{g}, \mathbb{R}\right) .
$$

This latter being the dual of the natural map

$$
\mathrm{H}_{2}\left(\Sigma_{g}, \mathbb{R}\right) \rightarrow \mathrm{H}_{2, \ell^{1}}\left(\Sigma_{g}, \mathbb{R}\right),
$$

we have that

$$
\left\langle\rho^{(2)}\left(\kappa_{\mathcal{X}}\right),\left[\Sigma_{g}\right]\right\rangle=\left\langle\rho_{\mathrm{b}}^{(2)}\left(\kappa_{\mathcal{X}}^{\mathrm{b}}\right),\left[\Sigma_{g}\right]_{\ell^{1}}\right\rangle_{\mathrm{b}},
$$

where $\left[\Sigma_{g}\right]_{\ell^{1}}$ denotes the image of $\left[\Sigma_{g}\right]$ under (5.5). Thus

$$
\left|\mathrm{T}_{\rho}\right| \leq \frac{1}{2 \pi}\left\|\rho_{\mathrm{b}}^{(2)}\left(\kappa_{\mathcal{X}}^{\mathrm{b}}\right)\right\|\left\|\left[\Sigma_{g}\right]\right\|_{\ell^{1}} .
$$


Recall now that the $\ell^{1}$-norm $\left\|\left[\Sigma_{g}\right]\right\|_{\ell^{1}}$ of the fundamental class is called the simplicial area of $\Sigma_{g}$ and, by [39],

$$
\left\|\left[\Sigma_{g}\right]\right\|_{\ell^{1}}=4 g-4 .
$$

This, together with the norm decreasing property of the pullback in bounded cohomology and the value of the norm of the Kähler class in Theorem 5.1, implies on the one hand the inequality in Proposition 3.1(3) and on the other the following

Proposition 5.3. Any maximal representation is a tight homomorphism.

The following general result about tight homomorphisms, together with the above proposition, implies part of Theorem 4.6.

Theorem 5.4 ([16], [66]). Let $\mathbf{G}$ be a semisimple real algebraic group, $H$ a locally compact group, and assume that the symmetric space $\mathcal{X}_{G}$ associated to $G:=\mathbf{G}(\mathbb{R})^{\circ}$ is Hermitian. Then for a tight homomorphism $\rho: H \rightarrow G$ the following holds:

(1) The Zariski closure $\mathbf{L}$ of the image $\rho(H)$ is reductive;

(2) the real reductive group $L:=\mathbf{L}(\mathbb{R})^{\circ}$ has compact centralizer in $G$; and

(3) the symmetric space $\mathcal{X}_{L} \subset \mathcal{X}_{G}$ associated to $L$ is Hermitian.

Notice that the totally geodesic embedding $\mathcal{X}_{L} \subset \mathcal{X}_{G}$ in (3) is not necessarily holomorphic. However, there is a notion of tight embedding for Hermitian symmetric spaces which parallels the one for homomorphisms.

Definition 5.5 ([16], [66]). Given a totally geodesic embedding

$$
f: \mathcal{Y} \rightarrow \mathcal{X}
$$

of Hermitian symmetric spaces, we say that $f$ is tight if

$$
\sup _{\Delta \subset \mathcal{Y}} \int_{\Delta} f^{*} \omega_{\mathcal{X}}=\sup _{\Delta \subset \mathcal{X}} \int_{\Delta} \omega_{\mathcal{X}}
$$

where the supremum is taken over all smooth triangles with geodesic sides.

This corresponds for the associated homomorphism

$$
\rho: H_{\mathcal{Y}} \rightarrow \operatorname{Isom}(\mathcal{X})^{\circ}
$$

where $H_{\mathcal{Y}}$ is an appropriate finite covering of $\operatorname{Isom}(\mathcal{Y})^{\circ}$, to be a tight homomorphism. With this terminology, the inclusion $\mathcal{X}_{L} \subset \mathcal{X}_{G}$ in Theorem 5.4(3) is a tight embedding.

Here are some examples of tight embeddings:

ExAmple 5.6. The homomorphism $\tau_{\mathcal{P}}: \mathrm{SU}(1,1)^{r} \rightarrow \mathcal{X}$ associated to a maximal polydisc $\mathcal{P} \subset \mathcal{X}$ is tight; evidently, the embedding $\mathcal{P} \subset \mathcal{X}$ is both holomorphic and tight. 
EXAMPLE 5.7. The irreducible representation $\rho_{2 n}: \operatorname{SL}(2, \mathbb{R}) \rightarrow \operatorname{Sp}(2 n, \mathbb{R})$ is tight, and the associated totally geodesic embedding of $\mathcal{H}_{\mathbb{R}}^{2} \rightarrow \mathcal{X}_{\mathrm{Sp}(2 n, \mathbb{R})}$ is a tight embedding which is holomorphic only if $n=1$.

ExAmple 5.8. The embedding $T \subset \mathcal{X}$ of a maximal tube type subdomain in $\mathcal{X}$ is tight and holomorphic.

ExAmple 5.9. If $\mathcal{Y}$ is an irreducible Hermitian symmetric space and $f: \mathcal{Y} \rightarrow \mathcal{X}$ is a totally geodesic embedding, then $f$ is tight if and only if

$$
f^{*} \omega_{\mathcal{X}}= \pm \frac{\mathrm{rk} \mathcal{X}}{\mathrm{rk \mathcal {Y }}} \omega_{\mathcal{Y}}
$$

EXAmple 5.10. The embedding $\mathcal{X}_{\mathrm{Sp}(V)} \rightarrow \mathcal{X}_{\mathrm{SU}\left(V_{\mathbb{C}}\right)}$ in Example 2.1.2 is tight and holomorphic.

Observe now the following simple

Proposition 5.11. Let $H, G$ be connected semisimple Lie groups with finite center and associated symmetric spaces of Hermitian type. If $\rho: \Gamma_{g} \rightarrow H$ is maximal and $\rho^{\prime}: H \rightarrow G$ is tight, then $\rho^{\prime} \circ \rho$ is maximal.

This, together with Examples 5.6 and 5.7 above justifies the maximality of the representations in Examples 3.9 and 3.10.

Notice that in general totally geodesic embeddings between bounded symmetric domains do not induce maps between the corresponding Shilov boundaries even if they are holomorphic. This is however something else that tight homomorphism can provide for us, namely

Theorem 5.12 ([16], [66]). Let $\mathcal{X}, \mathcal{Y}$ be Hermitian symmetric spaces and $f: \mathcal{Y} \rightarrow$ $\mathcal{X}$ a tight embedding with associated homomorphism $\rho: H_{\mathcal{Y}} \rightarrow \operatorname{Isom}(\mathcal{X})^{\circ}$ (see (5.6)). Then there exists a $\rho$-equivariant map

$$
\check{f}: \check{S}_{\mathcal{Y}} \rightarrow \check{S}_{\mathcal{X}}
$$

Remark that, since the Shilov boundary of a Hermitian symmetric space is a homogeneous space, if such $\rho$-equivariant map exists, it is unique (up to translations).

The above theorem allows us also to deduce in great generality the existence of boundary maps for tight homomorphisms. Let $\Lambda$ be a countable discrete group and let $\theta$ be a probability measure on $\Lambda$. Recall that a Poisson boundary of the pair $(\Lambda, \theta)$ is a measurable $\Lambda$-space $B$ with a quasiinvariant probability measure $\nu$ such that there exists an isometric isomorphism between the space of bounded $\theta$-harmonic functions

$$
\begin{aligned}
\mathcal{H}^{\infty}(\Lambda, \theta):=\{f: \Lambda \rightarrow \mathbb{R}: f \text { is bounded and } \\
\left.\qquad f(g)=\int_{\Lambda} f(g h) d \theta(h), \forall g \in \Lambda\right\}
\end{aligned}
$$


and the space $\mathrm{L}^{\infty}(B, \nu)$, given by the Poisson formula

$$
f(g)=\int_{B} \psi(g x) d \nu(x) .
$$

Although we shall not need it here, we recall that, under natural assumptions on the measure $\theta$, a Poisson boundary in fact exists even for locally compact second countable groups, [44].

An immediate consequence of the Poisson formula (5.7) is that the measure $\nu$ is $\theta$-stationary, that is $\theta * \nu=\nu$. Moreover, it will be essential for our purposes that the action of $\Lambda$ on the Poisson boundary $B$ is amenable with respect to the measure $\nu,[69]$.

ThEOREM 5.13. Let $\Lambda$ be a countable discrete group with probability measure $\theta$ and let $\mathbf{G}$ be a semisimple real algebraic group such that the symmetric space $\mathcal{X}$ associated to $G:=\mathbf{G}(\mathbb{R})^{\circ}$ is Hermitian. If $(B, \nu)$ is a Poisson boundary for $(\Lambda, \theta)$ and $\rho: \Lambda \rightarrow G$ is a tight homomorphism, then there exists a $\rho$-equivariant measurable map

$$
\varphi: B \rightarrow \check{S}_{\mathcal{X}}
$$

Proof. Let $\mathbf{L}$ be the Zariski closure of $\rho(\Lambda)$. By Theorem 5.4 the symmetric space $\mathcal{Y}$ associated to $L:=\mathbf{L}(\mathbb{R})^{\circ}$ is Hermitian symmetric and the embedding $\mathcal{Y} \rightarrow \mathcal{X}$ is tight, so that Theorem 5.12 implies the existence of a $\rho$-equivariant map $\check{f}$ between the corresponding Shilov boundaries

$$
\check{f}: \check{S}_{\mathcal{Y}} \rightarrow \check{S}_{\mathcal{X}} .
$$

Let $\mathbf{Q}<\mathbf{L}$ be a maximal parabolic subgroup defined over $\mathbb{R}$ such that $\check{S}_{\mathcal{Y}} \cong$ $\mathbf{L}(\mathbb{R}) / \mathbf{Q}(\mathbb{R})$, and let $\mathbf{P}<\mathbf{Q}$ be a minimal parabolic subgroup defined over $\mathbb{R}$ contained in $\mathbf{Q}$, so that we have an equivariant map

$$
\mathbf{L}(\mathbb{R}) / \mathbf{P}(\mathbb{R}) \rightarrow \mathbf{L}(\mathbb{R}) / \mathbf{Q}(\mathbb{R}) \cong \check{S}_{\mathcal{Y}}
$$

Since the action of $\Lambda$ on $(B, \nu)$ is amenable, there exists a $\rho$-equivariant measurable map

$$
\varphi_{0}: B \rightarrow \mathcal{M}^{1}(\mathbf{L}(\mathbb{R}) / \mathbf{P}(\mathbb{R}))
$$

where $\mathcal{M}^{1}(\mathbf{L}(\mathbb{R}) / \mathbf{P}(\mathbb{R}))$ denotes the space of probability measures on $\mathbf{L}(\mathbb{R}) / \mathbf{P}(\mathbb{R})$. Since $\rho: \Lambda \rightarrow L$ has Zariski dense image, the $\Lambda$-action on $\mathbf{L}(\mathbb{R}) / \mathbf{P}(\mathbb{R})$ is mean proximal (see [13, Theorem 7.3]): this, together with the fact that $\nu$ is $\theta$-stationary, implies that for $\nu$-a. e. $b \in B, \varphi_{0}(b)$ is a Dirac measure, thus providing a map

$$
\varphi_{0}: B \rightarrow \mathbf{L}(\mathbb{R}) / \mathbf{P}(\mathbb{R})
$$

which composed with the maps in (5.8) and (5.9) provides the required $\rho$-equivariant map. 
As an application, given a compact surface group $\Gamma_{g}$, choose a hyperbolization of $\Sigma_{g}$ and let $\Gamma$ be the realization of $\Gamma_{g}$ as a cocompact lattice in $\mathrm{PU}(1,1)$. Then $\Gamma$ acts naturally on $\mathbb{S}^{1}=\partial \mathcal{D}_{1,1}$ and, in fact, a theorem of Furstenberg asserts that there exists a probability measure $\theta$ on $\Gamma$ such that $\mathbb{S}^{1}$ with the Lebesgue measure $\lambda$ is a Poisson boundary of $(\Gamma, \theta)$.

Corollary 5.14. Let $\mathbf{G}$ be a semisimple real algebraic group such that the symmetric space $\mathcal{X}$ associated to $G:=\mathbf{G}(\mathbb{R})^{\circ}$ is Hermitian and let $\rho: \Gamma \rightarrow G$ be a tight embedding of a cocompact lattice $\Gamma<\mathrm{PU}(1,1)$. Then there exists a $\rho$-equivariant measurable map

$$
\varphi: \mathbb{S}^{1} \rightarrow \check{S}_{\mathcal{X}}
$$

REMARK 5.15. For technical purposes one can show that if $F \subset \check{S}_{\mathcal{X}}$ is the set of points which are not transverse to a given point in $\check{S}_{\mathcal{X}}$, and $\varphi$ is the map in Corollary 5.14 , the set $\varphi^{-1}(F)$ has Lebesgue measure zero in $\mathbb{S}^{1}$.

\section{Symplectic Anosov Structures}

We focus in this section on maximal representations into a symplectic group $\operatorname{Sp}(V)$. Let thus $\rho: \Gamma_{g} \rightarrow \operatorname{Sp}(V)$ be any representation. We choose a hyperbolization $\Sigma$ of $\Sigma_{g}$, and let $\Gamma<\mathrm{PU}(1,1)=\operatorname{Aut}\left(\mathcal{D}_{1,1}\right)^{\circ}$ be the resulting realization of $\Gamma_{g}$. From now on we consider $\rho$ as a representation of $\Gamma$. The geodesic flow $\tilde{g}_{t}$ on the unit tangent bundle $T^{1} \mathcal{D}_{1,1}$ gives rise to a flow $\tilde{g}_{t}{ }^{\rho}$ on the total space of the flat symplectic bundle $\widetilde{E}^{\rho}:=T^{1} \mathcal{D}_{1,1} \times V$ over $T^{1} \mathcal{D}_{1,1}$ commuting with the diagonal $\Gamma$-action given by $\gamma(u, x):=(\gamma u, \rho(\gamma) x)$ which hence descends to a flow $g_{t}^{\rho}$ on the quotient $E^{\rho}:=\Gamma \backslash\left(T^{1} \mathcal{D}_{1,1} \times V\right)$ which is a flat symplectic bundle over the unit tangent bundle $T^{1} \Sigma$. The projection

$$
p: E^{\rho} \rightarrow T^{1} \Sigma
$$

is then equivariant with respect to the $g_{t}^{\rho}$-action on $E^{\rho}$ and to the action of the geodesic flow $g_{t}$ on $T^{1} \Sigma$.

Let $\langle\cdot, \cdot\rangle: E^{\rho} \times{ }_{p} E^{\rho} \rightarrow \mathbb{R}$ be the symplectic form on $E^{\rho}$. A positive complex structure on the symplectic bundle is a continuous section

$$
J: T^{1} \Sigma \rightarrow \operatorname{End}\left(E^{\rho}\right)
$$

such that

(1) $J_{u}$ is a complex structure on the fiber $E^{\rho}(u)$, and

(2) the form $\langle\cdot, J \cdot\rangle$ is symmetric and positive definite in each fiber.

We denote by $\|\cdot\|: E^{\rho} \rightarrow \mathbb{R}_{+}$the resulting Euclidean norm, and by $\|\cdot\|_{u}$ its value on the fiber $E^{\rho}(u)$ above the point $u \in T^{1} \Sigma$. 
Observe that any symplectic bundle over a paracompact base admits a positive complex structure. A Lagrangian subbundle of a symplectic bundle is a subbundle such that each fiber is a Lagrangian subspace. With this terminology we have then the following

Theorem 6.1. Assume that $\rho: \Gamma \rightarrow \mathrm{Sp}(V)$ is a maximal representation. Then there is a $g_{t}^{\rho}$-invariant splitting

$$
E^{\rho}=E_{-}^{\rho} \oplus E_{+}^{\rho}
$$

into continuous Lagrangian subbundles, and there exist a positive complex structure $J$ and a constant $A>0$ such that

(1) $J$ interchanges $E_{-}^{\rho}$ and $E_{+}^{\rho}$, and

(2) for all $t \geq 0$,

$$
\left\|g_{t}^{\rho} \xi\right\| \leq e^{-A t}\|\xi\| \text { for all } \xi \in E_{+}^{\rho}
$$

and

$$
\left\|g_{-t}^{\rho} \xi\right\| \leq e^{-A t}\|\xi\| \text { for all } \xi \in E_{-}^{\rho} .
$$

This result has interesting consequences on the metric properties of a maximal representation. To describe them, as well as for convenience in the proofs in $\S 8$, we specify a left invariant metric on the symmetric space $\mathcal{X}_{\mathrm{Sp}(V)}$ associated to $\mathrm{Sp}(V)$. Recall that $\mathcal{X}_{\mathrm{Sp}(V)}$ is the set of complex structures $J$ on $V$ such that $\langle\cdot, J \cdot\rangle$ is symmetric and positive definite. Denoting by $q_{J}$ the corresponding Euclidean norm on $V$, and by $\|I d\|_{J_{1}, J_{2}}$ the norm of the identity map between $\left(V, q_{J_{1}}\right)$ and $\left(V, q_{J_{2}}\right)$, we set

$$
d\left(J_{1}, J_{2}\right):=|\ln |\left|I d\left\|_{J_{1}, J_{2}}|+| \ln \right\| I d \|_{J_{2}, J_{1}}\right| \quad J_{1}, J_{2} \in \mathcal{X}_{\mathrm{Sp}(V)} .
$$

Of course, this distance is equivalent to the $G$-invariant Riemannian distance on $\mathcal{X}_{\mathrm{Sp}(V)}$, but it is more convenient for our purposes.

The statement of the next corollary does not depend on the choice of a hyperbolization.

Corollary 6.2. Let $\rho: \Gamma_{g} \rightarrow \operatorname{Sp}(V)$ be a maximal representation, $J \in \mathcal{X}_{\mathrm{Sp}(V)}$ a basepoint and $\ell$ the word length on $\Gamma_{g}$. Then the orbit map

$$
\begin{aligned}
\rho_{J}: \Gamma_{g} & \rightarrow \mathcal{X}_{\mathrm{Sp}(V)} \\
\gamma & \mapsto \rho(\gamma) J
\end{aligned}
$$

is a quasiisometric embedding, that is there are constants $A, B>0$ such that for every $\gamma \in \Gamma$

$$
A^{-1} \ell(\gamma)-B \leq d(\rho(\gamma) J, J) \leq A \ell(\gamma)+B
$$


Essential in the proof of Theorem 6.1 is the existence of the boundary map obtained in Corollary 5.14 from the boundary $\mathbb{S}^{1}=\partial \mathcal{D}_{1,1}$ of the Poincaré disk into the space of Lagrangians $\mathcal{L}(V)$ which relates the Maslov cocycle (see $\S 7$ ) to the orientation cocycle on $\mathbb{S}^{1}$. A priori this map is only measurable, but as a consequence of the continuity of the splitting in Theorem 6.1, it turns out to be continuous. In fact, this map plays a role analogous to the one of hyperconvex curves in the study of the Hitchin component of $\operatorname{Hom}\left(\Gamma_{g}, \mathrm{SL}(n, \mathbb{R})\right)$ in [50].

Corollary 6.3. Let $\rho: \Gamma \rightarrow \operatorname{Sp}(V)$ be a maximal representation. Then there is a $\rho$-equivariant continuous injective map

$$
\varphi: \mathbb{S}^{1} \rightarrow \mathcal{L}(V)
$$

with rectifiable image.

\section{Bounded Cohomology at Use}

The definition of continuous bounded cohomology in $\S 5$ is not very useful from a practical point of view, as many natural cocycles of geometric origin are not continuous. The homological algebra approach developed in [19], [56], [12] and [11] allows us to overcome this obstacles in the usual way: as in the homological algebra approach to continuous cohomology, there are appropriate notions of coefficients modules, of relatively injective modules and of strong resolutions, that is resolutions with an appropriate homotopy operator. The underlying philosophy is that we need not restrict to the standard resolution in $\S 5$, but any resolution satisfying certain conditions will suffice to compute the bounded cohomology in a completely canonical way. More specifically, the prominent role played by proper actions in the case of continuous cohomology is played by amenable actions in the case of bounded continuous cohomology.

Theorem 7.1 (Burger-Monod [19], Monod [56]). Let $G$ be a locally compact second countable group and $(S, \nu)$ a regular amenable $G$-space. Then the continuous bounded cohomology of $G$ is isometrically isomorphic to the cohomology of the complex

$$
0 \longrightarrow \mathrm{L}_{\text {alt }}^{\infty}(S, \mathbb{R})^{G} \stackrel{d}{\longrightarrow} \mathrm{L}_{\text {alt }}^{\infty}\left(S^{2}, \mathbb{R}\right)^{G} \stackrel{d}{\longrightarrow} \ldots
$$

with the usual homogeneous coboundary operator.

Here $\mathrm{L}_{\text {alt }}^{\infty}\left(S^{n}, \mathbb{R}\right)$ denotes the subspace of $\mathrm{L}^{\infty}\left(S^{n}, \mathbb{R}\right)$ consisting of functions such that $f(s)=\operatorname{sign}(\sigma) f(\sigma(s))$ for all $s \in S^{n}$ and $\sigma$ any permutation of the coordinates.

Without getting into the details of the amenability of an action (for which we refer the reader to [70]), let us mention that the action of a group $\Lambda$ on the Poisson 
boundary $(B, \nu)$ relative to a probability measure $\theta$ is amenable, as well as the action of a connected semisimple Lie group $G$ on the quotient $G / P$ by a minimal parabolic subgroup $P<G$. So, for example, the action of a surface group $\Gamma_{g}$ on $\mathbb{S}^{1}$ via a hyperbolization is amenable, but if $\mathcal{X}$ is a Hermitian symmetric space the action of $\operatorname{Isom}(\mathcal{X})^{\circ}$ on the Shilov boundary $\breve{S}_{\mathcal{X}}$ is not, unless the symmetric space has real rank one.

If in addition to being amenable the action of $G$ on $(S, \nu)$ is mixing, that is the diagonal action on $(S \times S, \nu \times \nu)$ is ergodic, then any $G$-invariant measurable function on $S \times S$ must be essentially constant, and hence $\mathrm{L}_{\text {alt }}^{\infty}\left(S^{2}, \mathbb{R}\right)^{G}=0$. This, together with Theorem 7.1 implies the following

Corollary 7.2. Let $G$ be a locally compact second countable group and $(S, \nu)$ a regular amenable mixing $G$-space. If $\mathcal{Z L}_{\text {alt }}^{\infty}\left(S^{3}, \mathbb{R}\right)$ denotes the subspace of cocycles in $\mathrm{L}_{\text {alt }}^{\infty}\left(S^{3}, \mathbb{R}\right)$, then we have a canonical isometric isomorphism

$$
\mathrm{H}_{\mathrm{cb}}^{2}(G, \mathbb{R}) \cong \mathcal{Z} \mathrm{L}_{\mathrm{alt}}^{\infty}\left(S^{3}, \mathbb{R}\right)^{G} .
$$

ExAmple 7.3. Since the $\Gamma_{g^{-}}$action on $\mathbb{S}^{1}$ is amenable and mixing, then

$$
\mathrm{H}_{\mathrm{b}}^{2}\left(\Gamma_{g}, \mathbb{R}\right) \cong \mathcal{Z L}_{\mathrm{alt}}^{\infty}\left(\left(\mathbb{S}^{1}\right)^{3}, \mathbb{R}\right)^{\Gamma_{g}} .
$$

Likewise if $G$ is a connected semisimple Lie group and $P<G$ is a minimal parabolic, then

$$
\mathrm{H}_{\mathrm{cb}}^{2}(G, \mathbb{R}) \cong \mathcal{Z L}_{\mathrm{alt}}^{\infty}\left((G / P)^{3}, \mathbb{R}\right)^{G}
$$

On the one hand this shows immediately that in degree two continuous bounded cohomology is a Banach space, on the other it allows us to represent bounded cohomology classes via meaningful cocycles defined on boundaries.

From now on we shall apply these considerations to the symplectic group $G=$ $\operatorname{Sp}(V)$; for ease of notation, set $\operatorname{dim} V=2 n$. Following Kashiwara [53, $\S 1.5]$, we recall that the Maslov index $\beta_{n}$ of three Lagrangians $L_{1}, L_{2}, L_{3} \in \mathcal{L}(V)$ is defined as the index $\beta_{n}\left(L_{1}, L_{2}, L_{3}\right) \in \mathbb{Z}$ of the quadratic form

$$
\begin{aligned}
L_{1} \oplus L_{2} \oplus L_{3} & \longrightarrow \mathbb{R} \\
\left(x_{1}, x_{2}, x_{3}\right) & \mapsto\left\langle x_{1}, x_{2}\right\rangle+\left\langle x_{2}, x_{3}\right\rangle+\left\langle x_{3}, x_{1}\right\rangle .
\end{aligned}
$$

The function $\beta_{n}: \mathcal{L}(V)^{3} \rightarrow \mathbb{Z}$ is a cocycle which takes integer values in the interval $[-n, n]$; more specifically, on the space $\mathcal{L}(V)^{(3)}$ of triples of Lagrangians which are pairwise transverse, its set of values is $\{-n,-n+2, \ldots, n-2, n\}$, and each fiber of $\beta_{n}$ is precisely an open $\operatorname{Sp}(V)$-orbit. Remark also that $\beta_{1}$ is nothing but the orientation cocycle on $\mathbb{S}^{1}$.

The space $\mathcal{F}(V)$ of complete isotropic flags is a homogeneous space of $\operatorname{Sp}(V)$ with a minimal parabolic subgroup as stabilizer, and therefore the $\operatorname{Sp}(V)$-action 
on $\mathcal{F}(V)$ is amenable. Let

$$
\operatorname{pr}: \mathcal{F}(V) \rightarrow \mathcal{L}(V)
$$

be the projection

$$
\operatorname{pr}\left(\{0\} \subsetneq V_{1} \subsetneq \cdots \subsetneq V_{n}\right):=V_{n} .
$$

With these notations we have:

Proposition 7.4. The map

$$
\beta_{n} \circ \mathrm{pr}^{3}: \mathcal{F}(V)^{3} \rightarrow \mathbb{Z}
$$

is a bounded $\operatorname{Sp}(V)$-invariant alternating cocycle such that $\pi\left(\beta_{n} \circ \mathrm{pr}^{3}\right)$ corresponds to the bounded Kähler class $\kappa_{\mathrm{Sp}(V)}^{\mathrm{b}} \in \mathrm{H}_{\mathrm{cb}}^{2}(\mathrm{Sp}(V, \mathbb{R})$ under the isometric isomorphism in Corollary 7.2. In particular

$$
\left\|\kappa_{\mathrm{Sp}(V)}^{\mathrm{b}}\right\|=\left\|\pi\left(\beta_{n} \circ \mathrm{pr}^{3}\right)\right\|_{\infty}=\pi n .
$$

Of course the drawback of the acquired freedom in going from continuous functions to $\mathrm{L}^{\infty}$ functions - or, more specifically, function classes - is that now the implementation of the pullback of a bounded cohomology class cannot be done mindlessly as before, since pullbacks even via continuous maps do not define, in general, a well defined equivalence class of measurable functions. However, the situation is much simpler in our case, given that our class admits as a representative the Borel function in (7.1) for which the cocycle identity holds everywhere. The following important result is a particular case of a general phenomenon for which we refer the reader to [12].

Theorem 7.5. Let $\Gamma_{g} \rightarrow \operatorname{Sp}(V)$ be a homomorphism, and assume that there exists a $\rho$-equivariant measurable map

$$
\varphi: \mathbb{S}^{1} \rightarrow \mathcal{L}(V),
$$

where $\Gamma_{g}$ acts on $\mathbb{S}^{1}$ via a hyperbolization. Then the pullback

$$
\rho_{\mathrm{b}}^{(2)}\left(\kappa_{\mathrm{Sp}(V)}^{\mathrm{b}}\right) \in \mathrm{H}_{\mathrm{cb}}^{2}\left(\Gamma_{g}, \mathbb{R}\right)
$$

is represented by the cocycle $\pi\left(\beta_{n} \circ \varphi^{3}\right):\left(\mathbb{S}^{1}\right)^{3} \rightarrow \mathbb{R}$ defined by

$$
(x, y, z) \mapsto \pi \beta_{n}(\varphi(x), \varphi(y), \varphi(z)) .
$$

Now we succeeded in implementing the pullback in a rather effective way, but we find ourselves in the infinite dimensional Banach space $\mathrm{H}_{\mathrm{b}}^{2}\left(\Gamma_{g}, \mathbb{R}\right)$. To size things down again, we shall need to make use of the transfer map.

Choose a hyperbolization of $\Sigma_{g}$ and let as before $\Gamma$ be the realization of $\Gamma_{g}$ as a cocompact lattice in $\mathrm{PU}(1,1)$. Inspired by Example 7.3 and by the fact that

$$
\mathrm{H}_{\mathrm{cb}}^{2}(\mathrm{PU}(1,1), \mathbb{R}) \cong \mathcal{Z L}_{\mathrm{alt}}^{\infty}\left(\left(\mathbb{S}^{1}\right)^{3}, \mathbb{R}\right)^{\mathrm{PU}(1,1)},
$$


define a transfer map

$$
t: \mathrm{L}^{\infty}\left(\left(\mathbb{S}^{1}\right)^{3}, \mathbb{R}\right)^{\Gamma} \rightarrow \mathrm{L}^{\infty}\left(\left(\mathbb{S}^{1}\right)^{3}, \mathbb{R}\right)^{\mathrm{PU}(1,1)}
$$

by

$$
t f(x, y, z):=\int_{\Gamma \backslash \mathrm{PU}(1,1)} f(g x, g y, g z) d \mu(g),
$$

where $\mu$ is the $\mathrm{PU}(1,1)$-invariant probability measure on $\Gamma \backslash \mathrm{PU}(1,1)$. Since by Proposition 7.4

$$
\mathrm{H}_{\mathrm{cb}}^{2}(\operatorname{Sp}(V), \mathbb{R}) \cong \mathbb{R} \cdot\left(\beta_{n} \circ \mathrm{pr}^{3}\right)
$$

and

$$
\mathrm{H}_{\mathrm{cb}}^{2}(\mathrm{PU}(1,1), \mathbb{R}) \cong \mathbb{R} \cdot \beta_{1},
$$

composition of the pullback implemented as in Theorem 7.5 followed by the transfer map in cohomology

$$
\mathrm{H}_{\mathrm{cb}}^{2}(\mathrm{Sp}(V), \mathbb{R}) \stackrel{\rho_{\mathrm{b}}^{(2)}}{\longrightarrow} \mathrm{H}_{\mathrm{b}}^{2}(\Gamma, \mathbb{R}) \stackrel{t^{(2)}}{\longrightarrow} \mathrm{H}_{\mathrm{cb}}^{2}(\mathrm{PU}(1,1), \mathbb{R})
$$

implies that there exists a constant $c \geq 0$ such that for almost all $x, y, z \in \mathbb{S}^{1}$

$$
\int_{\Gamma \backslash \mathrm{PU}(1,1)} \beta_{n}(\varphi(g x), \varphi(g y), \varphi(g z)) d \mu(g)=c \beta_{1}(x, y, z) .
$$

An analogous composition of maps as in (7.2) in ordinary cohomology and their interplay via the comparison map which for $\operatorname{Sp}(V)$ and $\mathrm{PU}(1,1)$ are isomorphisms [19], allow us to explicit the constant $c$ in $(7.3)$ as explained in $[43, \S 3]$ in the context of Matsumoto's theorem.

Theorem 7.6. Let $\rho: \Gamma_{g} \rightarrow \operatorname{Sp}(V)$ be a homomorphism, $\Gamma<\mathrm{PU}(1,1)$ a hyperbolization of $\Gamma_{g}$, and assume that there exists a $\rho$-equivariant measurable map $\varphi: \mathbb{S}^{1} \rightarrow \mathcal{L}(V)$. Then for almost every $x, y, z \in \mathbb{S}^{1}$

$$
\int_{\Gamma \backslash \mathrm{PU}(1,1)} \beta_{n}(\varphi(g x), \varphi(g y), \varphi(g z)) d \mu(g)=\frac{\mathrm{T}_{\rho}}{\left|\chi\left(\Sigma_{g}\right)\right|} \beta_{1}(x, y, z) .
$$

Observe that if either $\rho(\Gamma)$ is Zariski dense or $\rho$ is tight, such a measurable $\Gamma$-equivariant map exists. The following corollary is then immediate from Theorem 5.13 and Theorem 7.6.

Corollary 7.7. Let $\rho: \Gamma \rightarrow \operatorname{Sp}(V)$ be a maximal representation. Then there exists a $\rho$-equivariant measurable map $\varphi: \mathbb{S}^{1} \rightarrow \mathcal{L}(V)$ and it satisfies

$$
\beta_{n}(\varphi(x), \varphi(y), \varphi(z))=n \beta_{1}(x, y, z)
$$

for almost every $x, y, z \in \mathbb{S}^{1}$. 


\section{Symplectic Anosov Structures: Proofs}

In this section we prove the results stated in $\S 6$. These proofs rest entirely on Corollary 7.7 and are otherwise independent of the machinery used to establish Corollary 7.7.

8.1. The Geometry of Triples of Lagrangians. Here we collect a few basic facts about the Maslov cocycle. Our reference is $[53, \S 1.5]$.

The space $\mathcal{L}(V)^{(3)}$ of triples of pairwise transverse Lagrangians decomposes as a union $\sqcup_{j=0}^{n} \mathcal{O}_{n-2 j}$ of $(n+1)$ open $\operatorname{Sp}(V)$-orbits such that $\mathcal{O}_{n-2 j}$ is the level set of $\beta_{n}$ where $\beta_{n}$ takes the value $n-2 j$.

The maximal value $n$ is special in that, if $L_{1}, L_{2}, L_{3}$ are not pairwise transverse, then $\left|\beta_{n}\left(L_{1}, L_{2}, L_{3}\right)\right|<n$, [53, Proposition 1.5.10]. Thus we observe that

$$
\text { if } \beta_{n}\left(L_{1}, L_{2}, L_{3}\right)= \pm n,
$$

then $L_{1}, L_{2}, L_{3}$ are pairwise transverse.

Given $L_{1}, L$ and $L_{3}$ with $L_{1}$ and $L$ transverse to $L_{3}$, consider the linear map $T_{13}: L_{1} \rightarrow L_{3}$ defined by

$$
L=\left\{\ell_{1}+T_{13}\left(\ell_{1}\right): \ell_{1} \in L_{1}\right\}
$$

and the quadratic form $Q_{L}^{L_{1}, L_{3}}: L_{1} \rightarrow \mathbb{R}$ defined by

$$
Q_{L}^{L_{1}, L_{3}}(x):=\left\langle x, T_{13} x\right\rangle .
$$

Let now

$$
t\left(L_{3}\right):=\left\{L \in \mathcal{L}(V): L \cap L_{3}=\{0\}\right\}
$$

and let $\mathcal{Q}\left(L_{1}\right)$ be the space of quadratic forms on $L_{1}$. Then we have a diffeomorphism

$$
\begin{aligned}
t\left(L_{3}\right) & \rightarrow \mathcal{Q}\left(L_{1}\right) \\
L & \mapsto Q_{L}^{L_{1}, L_{3}}
\end{aligned}
$$

and moreover (see [53, Lemma 1.5.4])

$$
\beta_{n}\left(L_{1}, L, L_{3}\right)=\operatorname{sign}\left(Q_{L}^{L_{1}, L_{3}}\right) .
$$

If $\tau:=\left(L_{1}, L_{2}, L_{3}\right)$ is a triple of pairwise transverse Lagrangians, we have an endomorphism $J(\tau)$ of $V=L_{1} \oplus L_{3}$ given in block form by

$$
J(\tau):=\left(\begin{array}{cc}
0 & -T_{31} \\
T_{13} & 0
\end{array}\right)
$$

which, since $J(\tau)^{2}=-I d$, defines a complex structure on $V$; moreover $\langle\cdot, J(\tau) \cdot\rangle$ is symmetric and the associated quadratic form $q_{J(\tau)}$ is the orthogonal direct 


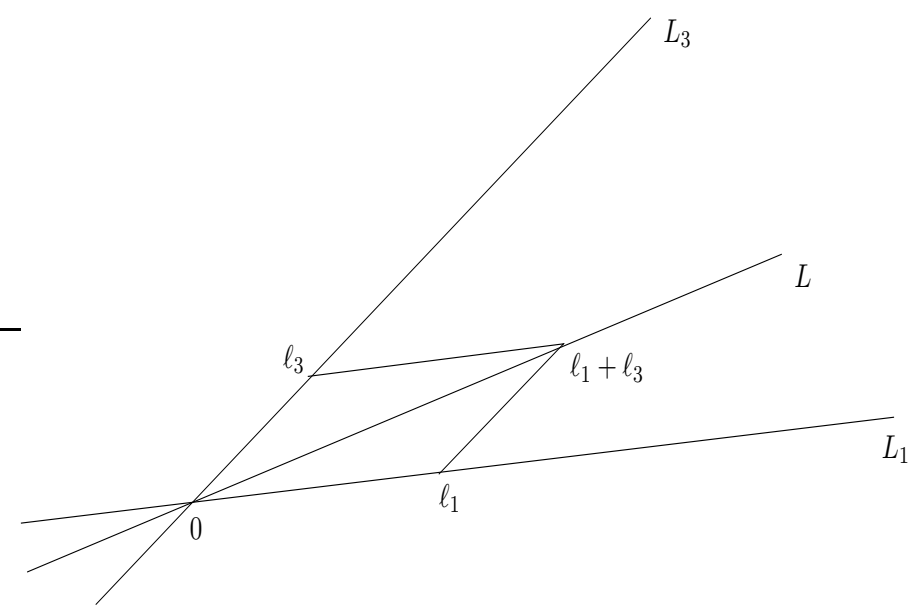

Figure 2. Let $L_{1}$ and $L$ be transverse to $L_{3}$. Then the vector $\ell_{3} \in L_{3}$ is the image of the vector $\ell_{1} \in L_{1}$ under the isomorphism $T_{13}: L_{1} \rightarrow L_{3}$ defined by $L$. The value at $\ell_{1}$ of the quadratic form $Q_{L}^{L_{1}, L_{3}}$ on $L_{1}$ based at $L_{3}$ and induced by $L$ measures the signed area of the parallelogram with vertices $0, \ell_{1}, \ell_{1}+\ell_{3}, \ell_{3}$. Moreover, if also $L_{1}$ and $L$ are transverse, then $Q_{L}^{L_{1}, L_{3}}\left(\ell_{1}\right)=-Q_{L}^{L_{3}, L_{1}}\left(\ell_{3}\right)$, where $\ell_{1}$ and $\ell_{3}$ are related as above.

sum of $Q_{L_{2}}^{L_{1}, L_{3}}$ on $L_{1}$ and $-Q_{L_{2}}^{L_{3}, L_{1}}$ on $L_{3}$ (see Figure 2); in particular $q_{J(\tau)}$ has signature

$$
2 \beta_{n}\left(L_{1}, L_{2}, L_{3}\right)=\operatorname{sign}\left(Q_{L_{2}}^{L_{1}, L_{3}}\right)-\operatorname{sign}\left(Q_{L_{2}}^{L_{3}, L_{1}}\right) .
$$

If now $\mathcal{L}(V)_{\text {max }}^{3}$ denotes the set of triples $\tau$ for which $\beta_{n}(\tau)=n$, we obtain an $\operatorname{Sp}(V)$-equivariant map

$$
\begin{aligned}
\mathcal{L}(V)_{\max }^{3} & \rightarrow \mathcal{X}_{\mathrm{Sp}(V)} \\
\tau & \longmapsto J(\tau)
\end{aligned}
$$

into the symmetric space $\mathcal{X}_{\mathrm{Sp}(V)}$ associated to $\operatorname{Sp}(V)$.

DeFinition 8.1. We say that a quadruple $\tau^{\prime}$ of Lagrangians is maximal if $\beta(\tau)=$ $n$ for any subtriple of Lagrangians $\tau$ taken in the same cyclic order as in $\tau^{\prime}$.

In particular (8.1) implies that a maximal quadruple consists of pairwise transverse Lagrangians. Finally we have the following important monotonicity property:

Lemma 8.2. Assume that the quadruple of Lagrangians $\left(L_{0}, L_{1}, L_{2}, L_{\infty}\right)$ is maximal. Then

$$
0<Q_{L_{1}}^{L_{0}, L_{\infty}}<Q_{L_{2}}^{L_{0}, L_{\infty}}
$$


and

$$
Q_{L_{1}, L_{0}}^{L_{\infty}}<Q_{L_{2}}^{L_{\infty}, L_{0}}<0
$$

Proof. For $\ell_{0} \in L_{0}$, let $\ell_{\infty}, \ell_{\infty}^{\prime} \in L_{\infty}$ with $\ell_{0}+\ell_{\infty} \in L_{1}$ and $\ell_{0}+\ell_{\infty}^{\prime} \in L_{2}$. Then

$$
\begin{aligned}
Q_{L_{2}}^{L_{0}, L_{\infty}}\left(\ell_{0}\right)-Q_{L_{1}}^{L_{0}, L_{\infty}}\left(\ell_{0}\right) & =\left\langle\ell_{0}, \ell_{\infty}^{\prime}-\ell_{\infty}\right\rangle \\
& =\left\langle\ell_{0}+\ell_{\infty}, \ell_{\infty}^{\prime}-\ell_{\infty}\right\rangle \\
& =Q_{L_{2}}^{L_{1}, L_{\infty}}\left(\ell_{0}+\ell_{\infty}\right),
\end{aligned}
$$

where the last equality follows from the fact that $\left(\ell_{0}+\ell_{\infty}\right) \in L_{1}, \ell_{\infty}^{\prime}-\ell_{\infty} \in L_{\infty}$, and their sum $\ell_{0}+\ell_{\infty}^{\prime} \in L_{2}$. Maximality of $\left(L_{1}, L_{2}, L_{\infty}\right)$ implies that $Q_{L_{2}}^{L_{1}, L_{\infty}}>0$, and maximality of $\left(L_{0}, L_{1}, L_{\infty}\right)$ implies that $Q_{L_{1}}^{L_{0}, L_{\infty}}>0$. Hence the assertion.

Notice that in the proof of Lemma 8.2 what was used is exactly the fact that the Lagrangians $L_{0}, L_{1}, L_{2}, L_{\infty}$ are pairwise transverse and that the triples $\left(L_{0}, L_{1}, L_{\infty}\right)$ and $\left(L_{1}, L_{2}, L_{\infty}\right)$ are maximal, which however, via the cocycle identity for $\beta_{n}$, is equivalent to the maximality of the quadruple $\left(L_{0}, L_{1}, L_{2}, L_{\infty}\right)$ (see the proof of Lemma 8.4).

8.2. Proofs of the Results in $\S$ 6. Let $\rho: \Gamma \rightarrow \operatorname{Sp}(V)$ be a maximal representation and let $\varphi: \mathbb{S}^{1} \rightarrow \mathcal{L}(V)$ be the $\rho$-equivariant measurable map given by Corollary 7.7. Paramount in the study of regularity properties of the map $\varphi$ is the closer analysis of its essential graph which we now introduce. Let $\lambda$ be the Lebesgue measure on $\mathbb{S}^{1}$. The essential graph $\mathcal{E}_{\varphi}$ of $\varphi$ is the closed subset $\mathcal{E}_{\varphi} \subset \mathbb{S}^{1} \times \mathcal{L}(V)$ which is the support of the pushforward of the measure $\lambda$ under the map

$$
\begin{aligned}
\mathbb{S}^{1} & \rightarrow \mathbb{S}^{1} \times \mathcal{L}(V) \\
x & \mapsto(x, \varphi(x)) .
\end{aligned}
$$

Here and in the sequel we shall often use the observation that

$$
\text { for almost every } x \in \mathbb{S}^{1},(x, \varphi(x)) \in \mathcal{E}_{\varphi} .
$$

Lemma 8.3. Let $\left(x_{1}, L_{1}\right),\left(x_{2}, L_{2}\right),\left(x_{3}, L_{3}\right) \in \mathcal{E}_{\varphi}$, and assume that

(1) $x_{1}, x_{2}, x_{3}$ are pairwise distinct, and

(2) $L_{1}, L_{2}, L_{3}$ are pairwise transverse.

Then $\beta_{n}\left(L_{1}, L_{2}, L_{3}\right)=n \beta_{1}\left(x_{1}, x_{2}, x_{3}\right)$.

Proof. We may assume that $\beta_{1}\left(x_{1}, x_{2}, x_{3}\right)=1$. Using that $\left(x_{i}, L_{i}\right) \in \mathcal{E}_{\varphi}$, Corollary 7.7 and the definition of essential graph imply that we may find sequences $L_{i}^{(k)}, i=1,2,3, k \in \mathbb{N}$, such that $\beta_{n}\left(L_{1}^{(k)}, L_{2}^{(k)}, L_{3}^{(k)}\right)=n$ and $\left(L_{1}^{(k)}, L_{2}^{(k)}, L_{3}^{(k)}\right)$ converges to $\left(L_{1}, L_{2}, L_{3}\right)$. In particular, $\left(L_{1}, L_{2}, L_{3}\right)$ is in the closure $\overline{\mathcal{O}_{n}}$ in $\mathcal{L}(V)^{3}$ 
of $\mathcal{O}_{n}$. Since on the other hand this triple belongs to $\sqcup_{j=0}^{n} \mathcal{O}_{n-2 j}$, observing that $\mathcal{O}_{k} \cap \overline{\mathcal{O}_{n}}=\emptyset$ for $k \neq n$, we conclude that $\left(L_{1}, L_{2}, L_{3}\right) \in \mathcal{O}_{n}$.

Notice now that any two (distinct) points $x_{1}, x_{2} \in \mathbb{S}^{1}$ determine an interval in $\mathbb{S}^{1}$, by defining

$$
\left(\left(x_{1}, x_{2}\right)\right):=\left\{t \in \mathbb{S}^{1}: \beta_{1}\left(x_{1}, t, x_{2}\right)=1\right\} .
$$

Lemma 8.4. Let $\left(x_{1}, L_{1}\right)$ and $\left(x_{2}, L_{2}\right) \in \mathcal{E}_{\varphi}$ with $x_{1} \neq x_{2}$. Then $L_{1}$ and $L_{2}$ are transverse.

Proof. Using Corollary 7.7, (8.6) and Remark 5.15 twice, we may choose $a \in\left(\left(x_{1}, x_{2}\right)\right)$ such that $(a, \varphi(a)) \in \mathcal{E}_{\varphi}$ and $\varphi(a)$ is transverse to $L_{1}, L_{2}$, and choose $b \in\left(\left(x_{2}, x_{1}\right)\right)$ so that $(b, \varphi(b)) \in \mathcal{E}_{\varphi}$ and $\varphi(b)$ is transverse to $\varphi(a), L_{1}, L_{2}$.

Applying the cocycle property of $\beta_{n}$ to the quadruple $\varphi(a), L_{2}, \varphi(b), L_{1}$, we have that

$$
\begin{aligned}
& \beta_{n}\left(L_{2}, \varphi(b), L_{1}\right)-\beta_{n}\left(\varphi(a), \varphi(b), L_{1}\right) \\
+ & \beta_{n}\left(\varphi(a), L_{2}, L_{1}\right)-\beta_{n}\left(\varphi(a), L_{2}, \varphi(b)\right)=0 ;
\end{aligned}
$$

since it follows from Lemma 8.3 that

$$
\beta_{n}\left(\varphi(a), \varphi(b), L_{1}\right)=n=\beta_{n}\left(\varphi(a), L_{2}, \varphi(b)\right),
$$

we obtain that

$$
\beta_{n}\left(L_{2}, \varphi(b), L_{1}\right)+\beta_{n}\left(\varphi(a), L_{2}, L_{1}\right)=2 n,
$$

which implies in turn that

$$
\beta_{n}\left(L_{2}, \varphi(b), L_{1}\right)=\beta_{n}\left(\varphi(a), L_{2}, L_{1}\right)=n .
$$

It follows hence from (8.1) that $L_{1}$ and $L_{2}$ are transverse.

From Lemmas 8.3 and 8.4 we deduce the following

Corollary 8.5. For $\left(x_{1}, L_{1}\right),\left(x_{2}, L_{2}\right),\left(x_{3}, L_{3}\right) \in \mathcal{E}_{\varphi}$ with $\left(x_{1}, x_{2}, x_{3}\right)$ pairwise distinct, we have

$$
\beta_{n}\left(L_{1}, L_{2}, L_{3}\right)=n \beta_{1}\left(x_{1}, x_{2}, x_{3}\right) .
$$

For the following, it will be convenient to define for $A \subset \mathbb{S}^{1}$ the "image of $A$ " by $\mathcal{E}_{\varphi}$

$$
F_{A}:=\left\{L \in \mathcal{L}(V): \text { there exists } a \in A \text { such that }(a, L) \in \mathcal{E}_{\varphi}\right\}
$$

which is closed if $A \subset \mathbb{S}^{1}$ is so. Now let us fix any two distinct points $x, y \in \mathbb{S}^{1}$. Lemma 8.6. The sets $\bar{F}_{((y, x))} \cap F_{\{x\}}$ and $\bar{F}_{((x, y))} \cap F_{\{x\}}$ both consist of one point.

Proof. Assume that there are $L_{0}, L_{0}^{\prime} \in \bar{F}_{((x, y))} \cap F_{\{x\}}$ and fix $L_{\infty} \in F_{\{y\}}$. By hypothesis, there are sequences $\left(x_{n}, L_{n}\right)$ and $\left(x_{n}^{\prime}, L_{n}^{\prime}\right)$ in $\mathcal{E}_{\varphi}$ with 
(1) $x_{n}, x_{n}^{\prime} \in((x, y))$, and $\lim x_{n}=\lim x_{n}^{\prime}=x$;

(2) $\lim L_{n}=L_{0}$ and $\lim L_{n}^{\prime}=L_{0}^{\prime}$.

By Lemma 8.4 all $L_{n}$ and $L_{n}^{\prime}$ are transverse to $L_{\infty}$ and we may thus use the diffeomorphism in (8.2)

$$
\begin{aligned}
t\left(L_{\infty}\right) & \rightarrow \mathcal{Q}\left(L_{0}\right) \\
L & \mapsto Q_{L}^{L_{0}, L_{\infty}}
\end{aligned}
$$

and study the situation in the model $\mathcal{Q}\left(L_{0}\right)$. Dropping the superscript $L_{0}, L_{\infty}$, we have that $\lim Q_{L_{n}}=Q_{L_{0}}=0$. For every $k \geq 1$, there is $N(k)$ such that $x_{n}^{\prime} \in\left(\left(x, x_{k}\right)\right)$ for all $n \geq N(k)$, and consequently $L_{0}, L_{n}^{\prime}, L_{k}, L_{\infty}$ is maximal; using Lemma 8.2, this implies that

$$
Q_{L_{0}}=0 \leq Q_{L_{n}^{\prime}} \leq Q_{L_{k}}
$$

and hence $\lim _{n} Q_{L_{n}^{\prime}}=0$. This shows that $\lim _{n} L_{n}^{\prime}=L_{0}$ and hence $L_{0}^{\prime}=L_{0}$.

According to Lemma 8.6, for every $x \in \mathbb{S}^{1}$ define

$$
\varphi_{+}(x) \in \bar{F}_{((y, x))} \cap F_{\{x\}} \text { and } \varphi_{-}(x) \in \bar{F}_{((x, y))} \cap F_{\{x\}} .
$$

From the definitions one deduces immediately the following

Corollary 8.7. The maps

$$
\varphi_{+}, \varphi_{-}: \mathbb{S}^{1} \rightarrow \mathcal{L}(V)
$$

defined above are respectively left and right continuous and strictly $\Gamma$-equivariant.

Now we turn to our symplectic bundle $E^{\rho}$ introduced in $\S 6$ and the study of the properties of the flow $g_{t}^{\rho}$. To define the Lagrangian splitting of $E^{\rho}$ we parametrize $T^{1} \mathcal{D}_{1,1}$ by the set $\left(\mathbb{S}^{1}\right)^{(3)}$ of distinct triples of points on $\mathbb{S}^{1}$, as follows: to a unit vector $u \in T^{1} \mathcal{D}_{1,1}$ based at $x$ associate the triple $\left(u_{-}, u_{0}, u_{+}\right) \in \mathbb{S}^{1}$, where $u_{-} \in \mathbb{S}^{1}$ and $u_{+} \in \mathbb{S}^{1}$ are respectively the initial and ending point of the geodesic $\left[u_{-}, u_{+}\right]$ determined by $u$, and $u_{0} \in \mathbb{S}^{1}$ is the endpoint of the geodesic perpendicular to $\left[u_{-}, u_{+}\right]$at $x \in \mathcal{D}_{1,1}$ and oriented in such a way that $u_{0} \in\left(\left(u_{-}, u_{+}\right)\right)$. Notice that as $u$ moves along the geodesic $\left[u_{-}, u_{+}\right]$in the positive direction, the point $u_{0}$ approaches $u_{+}$but the points $u_{-}, u_{+}$stay unchanged, so that the vector $g_{t} u$ corresponds to the triple $\left(u_{-}, u_{t}, u_{+}\right)$(see Figure 3$)$.

Let $\varphi_{-}, \varphi_{+}: \mathbb{S}^{1} \rightarrow \mathcal{L}(V)$ be respectively the right and left continuous $\Gamma$ equivariant map in Corollary 8.7. For every $u \in T^{1} \mathcal{D}_{1,1}$, since $u_{-} \neq u_{+}$, Lemma 8.4 implies that $\varphi_{-}\left(u_{-}\right)$and $\varphi_{+}\left(u_{+}\right)$define transverse and hence complementary Lagrangians

$$
V=\varphi_{-}\left(u_{-}\right) \oplus \varphi_{+}\left(u_{+}\right)
$$




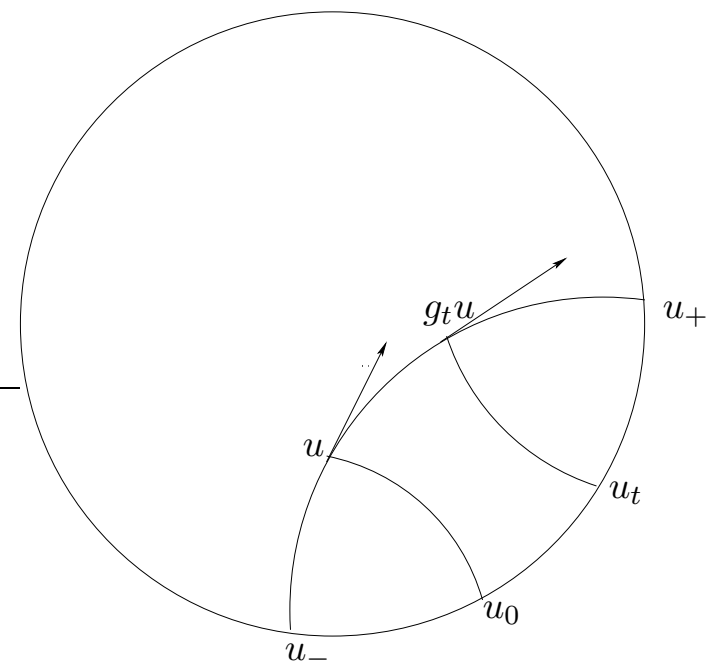

Figure 3 . The identification of $T^{1} \mathcal{D}_{1,1}$ with $\left(\mathbb{S}^{1}\right)^{(3)}$.

In this way we obtain a splitting of $\widetilde{E}^{\rho}$ into $\left(\tilde{g}_{t}^{\rho}\right)$-invariant Borel subbundles $\widetilde{E}^{\rho}=\widetilde{E}_{-}^{\rho} \oplus \widetilde{E}_{+}^{\rho}$ which descends to a $\left(g_{t}^{\rho}\right)$-invariant splitting

$$
E^{\rho}=E_{-}^{\rho} \oplus E_{+}^{\rho} .
$$

Using Corollary 8.5 we deduce that the triples $\left(\varphi_{-}\left(u_{-}\right), \varphi_{ \pm}\left(u_{t}\right), \varphi_{+}\left(u_{+}\right)\right)$are maximal for every $t$, so that we can associate to each of them complex structures $J\left(g_{t} u,+\right)$ and $J\left(g_{t} u,-\right)$ on $V$ as in (8.5), and hence positive quadratic forms $q_{J\left(g_{t} u,+\right)}$ and $q_{J\left(g_{t} u,-\right)}$, which thus give rise to two families $\|\cdot\|_{g_{t} u}^{+}$and $\|\cdot\|_{g_{t} u}^{-}$of Euclidean metrics on $E^{\rho}\left(g_{t} u\right)$, for $t \in \mathbb{R}, u \in T^{1} \mathcal{D}_{1,1}$.

Lemma 8.8. Let $p: E^{\rho} \rightarrow T^{1} \Sigma$ be the projection defined in (6.1) and, if $\xi \in E^{\rho}$, let $u:=p(\xi) \in T^{1} \Sigma$. Then

(1) For every $\xi \in E_{+}^{\rho}$

$$
\lim _{t \rightarrow+\infty}\left\|g_{t}^{\rho} \xi\right\|_{g_{t} u}^{+}=0 \text { monotonically, and }\left\|g_{-t}^{\rho} \xi\right\|_{g_{-t} u}^{ \pm} \geq\|\xi\|_{u}^{ \pm} \text {for all } t \geq 0 \text {. }
$$

(2) For every $\xi \in E_{-}^{\rho}$

$$
\lim _{t \rightarrow+\infty}\left\|g_{-t}^{\rho} \xi\right\|_{g_{-t} u}^{-}=0 \text { monotonically, and }\left\|g_{t}^{\rho} \xi\right\|_{g_{t} u}^{ \pm} \geq\|\xi\|_{u}^{ \pm}, \text {for all } t \geq 0 \text {. }
$$

Proof. We prove (1), as the proof of (2) is analogous. Working in $\widetilde{E}^{\rho}$ as we may, let $\xi \in \widetilde{E}^{\rho}, \xi=(u, v), v \in V$. Let $v \in \varphi_{+}\left(u_{+}\right)$. We use the Euclidean metrics $\|\cdot\|_{g_{t} u}^{+}$defined by the triple

$$
\left(\varphi_{-}\left(u_{-}\right), \varphi_{+}\left(u_{t}\right), \varphi_{+}\left(u_{+}\right)\right),
$$



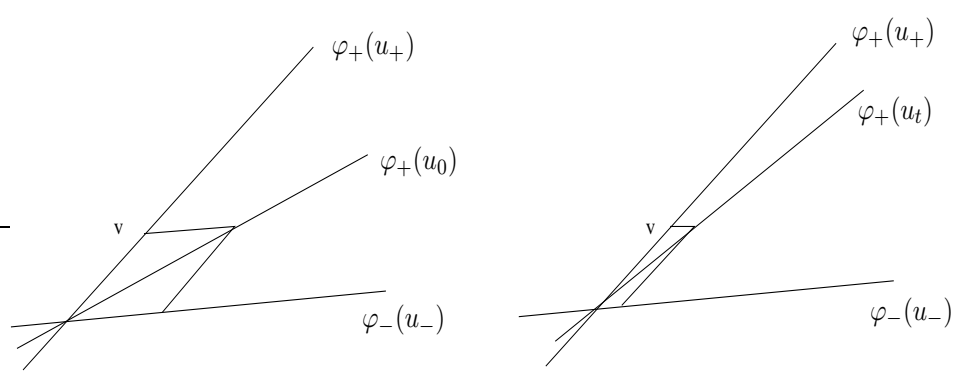

FiguRE 4.

that is

$$
\left\|\tilde{g}_{t}^{\rho} \xi\right\|_{g_{t} u}^{+}=\left|Q_{\varphi_{+}\left(u_{t}\right)}^{\varphi_{+}\left(u_{+}\right), \varphi_{-}\left(u_{-}\right)}(v)\right|
$$

which, since $\varphi_{+}$is left continuous and hence

$$
\lim _{t \rightarrow+\infty} \varphi_{+}\left(u_{t}\right)=\varphi_{+}\left(u_{+}\right),
$$

implies immediately that

$$
\lim _{t \rightarrow+\infty}\left\|\tilde{g}_{t}^{\rho} \xi\right\|_{g_{t} u}^{+}=0
$$

Monotonicity follows from Lemma 8.2. In fact, for every $0 \leq t_{1}<t_{2}$, the quadruple

$$
\left(\varphi_{-}\left(u_{-}\right), \varphi_{+}\left(u_{t_{1}}\right), \varphi_{+}\left(u_{t_{2}}\right), \varphi_{+}\left(u_{+}\right)\right)
$$

is maximal and hence Lemma 8.2 implies that

$$
\left\|\tilde{g}_{t_{2}}^{\rho} \xi\right\|_{g_{t_{2}} u}^{+} \leq\left\|\tilde{g}_{t_{1}}^{\rho} \xi\right\|_{g_{t_{1}} u}^{+}
$$

To prove the second statement in (1), observe that for $t \geq 0$ the quadruple

$$
\left(\varphi_{-}\left(u_{-}\right), \varphi_{+}\left(u_{-t}\right), \varphi_{+}\left(u_{0}\right), \varphi_{+}\left(u_{+}\right)\right)
$$

is maximal and hence Lemma 8.2 implies that

$$
\left\|\tilde{g}_{-t}^{\rho} \xi\right\|_{g_{-t} u}^{+}=\left|Q_{\varphi_{+}\left(u_{-t}\right)}^{\varphi_{+}\left(u_{+}\right), \varphi_{-}\left(u_{-}\right)}(v)\right| \geq\left|Q_{\varphi_{+}\left(u_{0}\right)}^{\varphi_{+}\left(u_{+}\right), \varphi_{-}\left(u_{-}\right)}(v)\right|=\|\xi\|_{u}^{+} .
$$

The statement for the metrics $\|\cdot\|_{g_{t} u}^{-}$follows analogously.

The metrics $\|\cdot\|_{u}^{+}$and $\|\cdot\|_{u}^{-}$are Borel metrics on the bundle $E^{\rho}$. Since the basis $T^{1} \Sigma$ is compact, any two continuous Euclidean metrics on $E^{\rho}$ are equivalent: we have then

Lemma 8.9. The metrics $\|\cdot\|_{u}^{+}$and $\|\cdot\|_{u}^{-}$are equivalent to a continuous metric.

This follows easily from the following two facts:

- The proper action of $\Gamma$ on $\left(\mathbb{S}^{1}\right)^{(3)}$ has compact quotient. 
- For any compact subset $C \subset\left(\mathbb{S}^{1}\right)^{(3)}$, the set of metrics

$$
\left\{\|\cdot\|_{u}^{ \pm}:\left(u_{-}, u_{0}, u_{+}\right) \in C\right\}
$$

is bounded.

Proof of Theorem 6.1. Fix a continuous Euclidean metric $\|\cdot\|$ on $E^{\rho}$. Then it follows from Lemmas 8.9 and 8.8 that

$$
E_{ \pm}^{\rho}:=\left\{\xi \in E^{\rho}: \lim _{t \rightarrow \pm \infty}\left\|g_{t}^{\rho} \xi\right\|=0\right\} .
$$

This implies by the following classical argument that the subbundles $E_{+}^{\rho}$ and $E_{-}^{\rho}$ are continuous. Let $u_{m}$ be a converging sequence in $T^{1} \Sigma$ with limit $u$, and let $F \subset E^{\rho}(u)$ be any accumulation point of

$$
\left\{E_{+}^{\rho}\left(u_{m}\right): m \geq 1\right\}
$$

in the Grassmann $n$-bundle of $E^{\rho}$. Let $\left\{m_{k}\right\}$ be a subsequence with $\lim _{k \rightarrow \infty} E_{+}^{\rho}$ $\left(u_{m_{k}}\right)=F$. For every $\xi \in F$ take $\xi_{k} \in E_{+}^{\rho}\left(u_{m_{k}}\right)$ with $\lim _{k \rightarrow \infty} \xi_{k}=\xi$. Then the function

$$
\begin{aligned}
\mathbb{R}^{+} & \rightarrow \mathbb{R}^{+} \\
t & \mapsto\left\|g_{t}^{\rho} \xi\right\|
\end{aligned}
$$

being a uniform limit on compacts of the sequence of functions $t \mapsto\left\|g_{t}^{\rho} \xi_{k}\right\|$ which vanish at infinity, vanishes at infinity as well, which implies that $\xi \in E_{+}^{\rho}(u)$ and hence $F \subseteq E_{+}^{\rho}(u)$; since both spaces have the same dimension, we conclude that $F=E_{+}^{\rho}(u)$. This shows continuity of the splitting.

This implies by the definition of $\widetilde{E}_{ \pm}^{\rho}$ that both maps $\varphi_{+}$and $\varphi_{-}$from $\mathbb{S}^{1}$ to $\mathcal{L}(V)$ are continuous. But this implies easily that $\varphi_{-}=\varphi_{+}$; we shall denote from now on by $\varphi$ this continuous $\Gamma$-equivariant map. This implies now the first assertion of Corollary 6.3.

We are thus in the following situation: for every $u \in T^{1} \mathcal{D}_{1,1}$, we have the splitting

$$
V=\varphi\left(u_{-}\right) \oplus \varphi\left(u_{+}\right), \quad u=\left(u_{-}, u_{0}, u_{+}\right)
$$

which gives rise to the splittings

$$
\widetilde{E}^{\rho}=\widetilde{E}_{-}^{\rho} \oplus \widetilde{E}_{+}^{\rho}
$$

and

$$
E^{\rho}=E_{-}^{\rho} \oplus E_{+}^{\rho}
$$

into continuous $\tilde{g}_{t}^{\rho}$ and $g_{t}^{\rho}$ invariant subbundles. We denote by $J(u) \in \mathcal{X}_{\mathrm{Sp}(V)}$ the complex structure associated to the triple

$$
\left(\varphi\left(u_{-}\right), \varphi\left(u_{0}\right), \varphi\left(u_{+}\right)\right)
$$


as in (8.5). It is now immediate that the map

$$
\begin{aligned}
T^{1} \mathcal{D}_{1,1} & \rightarrow \mathcal{X}_{\mathrm{Sp}(V)} \\
u & \longmapsto J(u)
\end{aligned}
$$

gives a positive complex structure $J$ of $E^{\rho}$ with the required properties (see (8.4)). Let $\|\cdot\|_{u}$ be the Euclidean metric on $E^{\rho}$ induced by the quadratic form $q_{J(u)}$.

In the notation of Lemma 8.8, we have $\|\cdot\|_{u}^{+}=\|\cdot\|_{u}^{-}=\|\cdot\|_{u}$ and hence for every $\xi \in E_{ \pm}^{\rho}$ with $p(\xi)=u$

$$
\lim _{t \rightarrow \infty}\left\|g_{ \pm t} \xi\right\|_{g_{ \pm t} u}=0 \text { monotonically. }
$$

We claim now that there exists $T>0$ such that for every $\xi \in E_{+}^{\rho}$,

$$
\left\|g_{t}^{\rho} \xi\right\|_{g_{t} u} \leq \frac{1}{2}\|\xi\|_{u} \quad \text { for } t \geq T .
$$

Indeed, if this were not the case, by Lemma 8.8 there would exist a sequence $\xi_{n} \in E_{+}^{\rho}$ and $T_{n} \rightarrow+\infty$ with $\left\|\xi_{n}\right\|=1$ and $\left\|g_{T_{n}}^{\rho} \xi_{n}\right\|_{g_{T_{n}} u_{n}}=\frac{1}{2}$. We may assume that $\xi_{n}$ converges to a point $\xi \in E_{+}^{\rho}$. Then the sequence of functions

$$
\begin{aligned}
\mathbb{R}^{+} & \longrightarrow \mathbb{R}^{+} \\
t & \mapsto\left\|g_{t}^{\rho} \xi_{n}\right\|_{g_{t} u_{n}}
\end{aligned}
$$

converges uniformly on compact sets to

$$
t \mapsto\left\|g_{t}^{\rho} \xi\right\|_{g_{t} u} .
$$

But, by monotonicity, we have that

$$
\left\|g_{t}^{\rho} \xi_{n}\right\|_{g_{t} u_{n}} \geq \frac{1}{2}, \quad \text { for } t \in\left[0, T_{n}\right]
$$

and since $T_{n} \rightarrow+\infty$, we deduce that

$$
\left\|g_{t}^{\rho} \xi\right\|_{g_{t} u} \geq \frac{1}{2} \quad \text { for all } t \geq 0,
$$

which contradicts the fact that $\xi \in E_{+}^{\rho}$. Applying the inequality

$$
\left\|g_{T}^{\rho} \xi\right\| \leq \frac{1}{2}\|\xi\|
$$

to $n T$, for $n \in \mathbb{N}$, we obtain the exponential decay.

Proof of Corollary 6.2. The proof will rely on the metric properties of the map defined in (8.7).

Fix a unit tangent vector $v \in T^{1} \mathcal{D}_{1,1}$ based at $0 \in \mathcal{D}_{1,1}$ and let $J_{0}:=J(v) \in$ $\mathcal{X}_{\mathrm{Sp}(V)}$ be the corresponding complex structure on $V$. Observe first of all that $d\left(J_{0}, \rho(\gamma) J_{0}\right)$ is bounded above linearly by the word length $\ell(\gamma)$ of $\gamma$, as an argument by recurrence on $\ell(\gamma)$ easily shows. In order to show the lower bound, we shall use the contraction-dilation property of the Anosov flow in Theorem 6.1(2). 
The essential step is estimating the distance in $\mathcal{X}_{\mathrm{Sp}(V)}$ between $J(u)$ and $J\left(g_{t} u\right)$, given by

$$
d\left(J(u), J\left(g_{t} u\right)\right)=|\ln |\left|I d\left\|_{J(u), J\left(g_{t} u\right)}|+| \ln || I d\right\|_{J\left(g_{t} u\right), J(u)}\right|,
$$

for any $u \in T^{1} \mathcal{D}_{1,1}$ and any $t \geq 0(\operatorname{see} \S 6)$.

For $x \in \varphi\left(u_{-}\right)$, applying Theorem 6.1 , we have that

$$
q_{J\left(g_{t} u\right)}(x) \geq e^{2 A t} q_{J(u)}(x)
$$

and likewise for $x \in \varphi\left(u_{+}\right)$

$$
q_{J\left(g_{t} u\right)}(x) \leq e^{-2 A t} q_{J(u)}(x) .
$$

These inequalities, together with the fact that $\varphi\left(u_{-}\right) \oplus \varphi\left(u_{+}\right)$is an orthogonal decomposition for both $q_{J(u)}$ and $q_{J\left(g_{t} u\right)}$, imply that

$$
\|I d\|_{J(u), J\left(g_{t} u\right)} \geq e^{A t}
$$

and

$$
\|I d\|_{J\left(g_{t} u\right), J(u)} \geq e^{A t},
$$

from which we deduce that

$$
d\left(J(u), J\left(g_{t} u\right)\right) \geq 2 A t .
$$

Let now $\gamma \in \Gamma$ and let us choose $u \in T^{1} \mathcal{D}_{1,1}$ to be the tangent vector at $0 \in \mathcal{D}_{1,1}$ to the geodesic segment connecting 0 to $\gamma 0$ and let $t=d(0, \gamma 0)$. Applying (8.8) to this situation and observing that $g_{t} u=\gamma u$, we get that

$$
d(J(u), \rho(\gamma) J(u)) \geq 2 A d(0, \gamma 0)
$$

and hence

$$
d\left(J_{0}, \rho(\gamma) J_{0}\right) \geq 2 A d(0, \gamma 0)-2 C,
$$

where

$$
C:=\sup \left\{d\left(J\left(w_{1}\right), J\left(w_{2}\right)\right): w_{1}, w_{2} \text { are based at } 0\right\} .
$$

Finally, $d(0, \gamma 0)$ is bounded linearly below in terms of $\ell(\gamma)$, as follows from the Milnor-Svarc lemma.

Proof of Corollary 6.3. The injectivity of the $\Gamma$-equivariant continuous map

$$
\varphi: \mathbb{S}^{1} \rightarrow \mathcal{L}(V)
$$

obtained in the proof of Theorem 6.1, follows for instance from Corollary 7.7 because of continuity. So we finally turn to the proof of the rectifiability of the image of $\varphi$. For this we shall put to use the $\operatorname{Sp}(V)$-invariant causal structure on $\mathcal{L}(V)$. 
Let us fix $a \neq b \in \mathbb{S}^{1}$, let $L_{0}:=\varphi(a)$ and $L_{\infty}:=\varphi(b)$, so that on $\mathbb{S}^{1} \backslash\{b\}, \varphi$ takes values in $t\left(L_{\infty}\right)$. Composing the restriction of $\varphi$ to $\mathbb{S}^{1} \backslash\{b\}$ with the usual diffeomorphism

$$
\begin{aligned}
t\left(L_{\infty}\right) & \rightarrow \mathcal{Q}\left(L_{0}\right) \\
L & \mapsto Q_{L}^{L_{0}, L_{\infty}},
\end{aligned}
$$

gives rise to a continuous map

$$
c: \mathbb{S}^{1} \backslash\{b\} \rightarrow \mathcal{Q}\left(L_{0}\right)
$$

whose restriction to the interval $((a, b))$ has the following properties:

(1) it takes values in the cone $\mathcal{Q}^{+}\left(L_{0}\right)$ of positive definite quadratic forms, and

(2) for every $t_{1}, t_{2} \in((a, b))$ such that $a, t_{1}, t_{2}, b$ are in positive cyclic order, $c\left(t_{2}\right)-c\left(t_{1}\right) \in \mathcal{Q}^{+}\left(L_{0}\right)$.

Fixing a scalar product on $L_{0}$, we can identify $\mathcal{Q}\left(L_{0}\right)$ with the space $\operatorname{Sym}\left(L_{0}\right)$ of symmetric endomorphisms of $L_{0}$ and $\mathcal{Q}^{+}\left(L_{0}\right)$ with the cone $\operatorname{Sym}^{+}\left(L_{0}\right)$ of positive definite ones. On $\operatorname{Sym}\left(L_{0}\right)$ we have a natural scalar product

$$
\langle\langle A, B\rangle\rangle:=\operatorname{tr} A B
$$

and we have that for every $A, B \in \operatorname{Sym}^{+}\left(L_{0}\right)$

$$
\langle\langle A, B\rangle\rangle>0 \text {, }
$$

that is $\operatorname{Sym}^{+}\left(L_{0}\right)$ is an open convex acute cone. The assertion then follows from the following general fact

Lemma 8.10. Let $C \subset E$ be an open convex acute cone in an Euclidean space and let $f:[0,1] \rightarrow C$ be a continuous map such that for every $t_{1}<t_{2}$,

$$
f\left(t_{2}\right)-f\left(t_{1}\right) \in C .
$$

Then $f$ is of finite length.

Proof. Fix $e \in C$. We claim that since $C$ is acute

$$
k:=\inf _{x \in C} \frac{\langle\langle x, e\rangle\rangle}{\|x\|}>0 .
$$

Indeed, otherwise there is a nonzero $x \in \bar{C}$ such that $\langle\langle x, e\rangle\rangle=0$. On the other hand, since $C$ is open, for $s<0$ and $|s|$ small enough we have that

$$
e^{\prime}:=s x+(1-s) e \in C,
$$

which implies that $\left\langle\left\langle e^{\prime}, x\right\rangle\right\rangle<0$ and contradicts the fact that $\langle\langle u, v\rangle\rangle \geq 0$ for all $u, v \in \bar{C}$. 
Let $0 \leq s<t \leq 1$; then $f(t)-f(s) \in C$ and applying the claim, we obtain:

$$
\|f(t)-f(s)\| \leq \frac{1}{k}\langle f(t)-f(s), e\rangle .
$$

Given any subdivision $0=t_{0}<t_{1}<\cdots<t_{n-1}<t_{n}=1$ of the interval $[0,1]$, we deduce that

$$
\sum_{i=1}^{n}\left\|f\left(t_{i}\right)-f\left(t_{i-1}\right)\right\| \leq \frac{1}{k} \sum_{i=1}^{n}\left\langle f\left(t_{i}\right)-f\left(t_{i-1}\right), e\right\rangle=\frac{\langle f(1)-f(0), e\rangle}{k}
$$

which proves that $f$ is rectifiable.

\section{REFERENCES}

1. Ch. Bavard, Longueur stable des commutateurs, Enseign. Math. (2) 37 (1991), no. 1-2, 109-150.

2. M. Bestvina and K. Fujiwara, Bounded cohomology of subgroups of mapping class groups, Geom. Topol. 6 (2002), 69-89 (electronic).

3. P. Biran, M. Entov, and L. Polterovich, Calabi quasimorphisms for the symplectic ball, Commun. Contemp. Math. 6 (2004), no. 5, 793-802.

4. S. B. Bradlow, O. García Prada, and P. B. Gothen, Surface group representations in $\mathrm{PU}(p, q)$ and Higgs bundles, J. Diff. Geom. 64 (2003), no. 1, 111-170.

5.

6. S. B. Bradlow, O. García-Prada, and P. Gothen, Moduli spaces of holomorphic triples over compact Riemann surfaces, Math. Ann. 328 (2004), no. 1-2, 299-351.

7. S. B. Bradlow, O. García-Prada, and P. B. Gothen, Representations of surface groups in the general linear group, Proceedings of the XII Fall Workshop on Geometry and Physics, Publ. R. Soc. Mat. Esp., vol. 7, R. Soc. Mat. Esp., Madrid, 2004, pp. 83-94.

8. R. Brooks, Some remarks on bounded cohomology, Riemann surfaces and related topics: Proceedings of the 1978 Stony Brook Conference (State Univ. New York, Stony Brook, N.Y., 1978) (Princeton, N.J.), Ann. of Math. Stud., vol. 97, Princeton Univ. Press, 1981, pp. 53-63.

9. R. Brooks and C. Series, Bounded cohomology for surface groups, Topology 23 (1984), no. 1, 29-36.

10. M. Bucher, Boundedness of characteristic classes for flat bundles, Ph.D. Thesis, ETH, 2004.

11. M. Burger and A. Iozzi, Bounded cohomology and deformation rigidity in hyperbolic geometry, preprint, arXiv:math.MG/0505069.

12. _ Boundary maps in bounded cohomology, Geom. Funct. Anal. 12 (2002), 281-292.

13. - Bounded Kähler class rigidity of actions on Hermitian symmetric spaces, Ann. Sci. École Norm. Sup. (4) 37 (2004), no. 1, 77-103.

14. M. Burger, A. Iozzi, and A. Wienhard, Hermitian symmetric spaces and Kähler rigidity, preprint.

15. _ Surface group representations with maximal Toledo invariant, preprint.

16. - Tight homomorphisms and Hermitian symmetric spaces, preprint.

17. _ Surface group representations with maximal Toledo invariant, C. R. Acad. Sci. Paris, Sér. I 336 (2003), 387-390.

18. M. Burger and N. Monod, Bounded cohomology of lattices in higher rank Lie groups, J. Eur. Math. Soc. 1 (1999), no. 2, 199-235.

19. Continuous bounded cohomology and applications to rigidity theory, Geom. Funct. Anal. 12 (2002), 219-280. 
20. E. Cartan, Sur les domaines bornés homogènes de l'espace de n variables complexes, Abh. Math. Sem. Hamburg 11 (1935), 116-162.

21. S. Choi and W. M. Goldman, Convex real projective structures on closed surfaces are closed, Proc. Amer. Math. Soc. 118 (1993), no. 2, 657-661.

22. J. L. Clerc and B. Ørsted, The Gromov norm of the Kaehler class and the Maslov index, Asian J. Math. 7 (2003), no. 2, 269-295.

23. P. Deligne, Espaces hermitiens symétriques, transcripted by J. Milne from Deligne's handwritten notes, Spring 1973. Available at http://www.jmilne.org/math/ under the title "Deligne's notes on Hermitian symmetric spaces".

24. A. Domic and D. Toledo, The Gromov norm of the Kähler class of symmetric domains, Math. Ann. 276 (1987), no. 3, 425-432.

25. J. L. Dupont, Bounds for characteristic numbers of flat bundles, Algebraic topology, Aarus 1978, Lecture Notes in Mathematics, vol. 763, Springer Verlag, 1979.

26. J. Eells and J. C. Wood, Restriction on harmonic maps of surfaces, Topology 15 (1976), 263-266.

27. M. Entov and L. Polterovich, Calabi quasimorphism and quantum homology, Int. Math. Res. Not. (2003), no. 30, 1635-1676.

28. D. B. A. Epstein and K. Fujiwara, The second bounded cohomology of word-hyperbolic groups, Topology 36 (1997), no. 6, 1275-1289.

29. V. V. Fock and A. B. Goncharov, Moduli spaces of convex projective structures on surfaces, preprint, arXiv:math.DG/0405348.

30. - Moduli spaces of local systems and higher Teichmüller theory, preprint, arXiv:math.AG/0311149.

31. J.-M. Gambaudo and É. Ghys, Commutators and diffeomorphisms of surfaces, Ergodic Theory Dynam. Systems 24 (2004), no. 5, 1591-1617.

32. O. García-Prada and I. Mundet i Riera, Representations of the fundamental group of a closed oriented surface in $\mathrm{Sp}(4, \mathbb{R})$, Topology 43 (2004), no. 4, 831-855.

33. E. Ghys, Groupes d'homéomorphismes du cercle et cohomologie bornée, The Lefschetz centennial conference, Part III, (Mexico City 1984), Contemp. Math., vol. 58, American Mathematical Society, RI, 1987, pp. 81-106.

34. W. M. Goldman, Discontinuous groups and the Euler class, Thesis, University of California at Berkeley, 1980.

35. W. M. Goldman, M. Kapovich, and B. Leeb, Complex hyperbolic manifolds homotopy equivalent to a riemann surface, Comm. Anal. Geom. 9 (2001), no. 1, 61-95.

36. W.M. Goldman, Topological components of spaces of representations, Invent. Math. 93 (1988), no. 3, 557-607.

37. P. B. Gothen, Components of spaces of representations and stable triples, Topology 40 (2001), no. 4, 823-850.

38. R. I. Grigorchuk, Some results on bounded cohomology, Combinatorial and geometric group theory (Edinburgh, 1993), London Math. Soc. Lecture Note Ser., vol. 204, Cambridge Univ. Press, Cambridge, 1995, pp. 111-163.

39. M. Gromov, Volume and bounded cohomology, Inst. Hautes Études Sci. Publ. Math. 56 (1982), 5-99.

40. Harish-Chandra, Representations of semisimple Lie groups. VI. Integrable and squareintegrable representations, Amer. J. Math. 78 (1956), 564-628.

41. L. Hernàndez Lamoneda, Maximal representations of surface groups in bounded symmetric domains, Trans. Amer. Math. Soc. 324 (1991), 405-420.

42. N. J. Hitchin, Lie groups and Teichmüller space, Topology 31 (1992), no. 3, 449-473. 
43. A. Iozzi, Bounded cohomology, boundary maps, and representations into $\mathrm{Homeo}_{+}\left(S^{1}\right)$ and $S U(1, n)$, Rigidity in Dynamics and Geometry, Cambridge, UK, 2000, Springer Verlag, 2002, pp. 237-260.

44. V. A. Kaimanovich, The Poisson boundary of a discrete group, preprint available at http://name.math.univ-rennes1.fr/vadim.kaimanovich/poifrm1w.ps.

45. H. Kneser, Die kleinste Bedeckungszahl innerhalb einer Klasse von Flächenabbildungen, Math. Ann. 103 (1930), 317-358.

46. A. Korányi, Function spaces on bounded symmetric domains, Analysis and Geometry on Complex Homogeneous Domains (J. Faraut et al., ed.), Progress in Mathematics, Birkhäuser, Boston, 2000, pp. 183-277.

47. D. Kotschick, Signatures, monopoles and mapping class groups, Math. Res. Lett. 5 (1998), no. 1-2, 227-234.

48. _ Quasi-homomorphisms and stable lengths in mapping class groups, Proc. Amer. Math. Soc. 132 (2004), no. 11, 3167-3175 (electronic).

49. What is. . a quasi-morphism?, Notices Amer. Math. Soc. 51 (2004), no. 2, 208-209.

50. F. Labourie, Anosov flows, surface groups and curves in projective space, preprint, arXiv:math.DG/0401230.

51. — Crossratios, surface groups, $S L(n, \mathbb{R})$ and $C^{1, h}\left(S^{1}\right) \rtimes \operatorname{Diff}^{h}\left(S^{1}\right)$, preprint, arXiv:math.DG/0502441.

52. J. F. Lafont and B. Schmidt, Simplicial volume of closed locally symmetric spaces of noncompact type, preprint, arXiv:math.DG/0504338.

53. G. Lion and M. Vergne, The Weil representation, Maslov index and theta series, Progress in Mathematics, vol. 6, Birkhäuser Boston, Mass., 1980.

54. J. Milnor, On the existence of a connection with curvature zero, Comment. Math. Helv. 32 (1958), 215-223.

55. I. Mineyev, Straightening and bounded cohomology of hyperbolic groups, Geom. Funct. Anal. 11 (2001), no. 4, 807-839.

56. N. Monod, Continuous bounded cohomology of locally compact groups, Lecture Notes in Math., no. 1758, Springer-Verlag, 2001.

57. I. I. Piateskii-Shapiro, Geometry of classical domains and theory of automorphic functions, Gordon and Breach, New York, 1969.

58. I. Satake, Algebraic structures of symmetric domains, Kanô Memorial Lectures, vol. 4, Iwanami Shoten, Tokyo, 1980.

59. R. Savage, The space of positive definite matrices and Gromov's invariant, Trans. Amer. Math. Soc. 274 (1982), no. 1, 241-261.

60. T. Soma, The third bounded cohomology and Kleinian groups, Topology and Teichmüller spaces (Katinkulta, 1995), World Sci. Publishing, River Edge, NJ, 1996, pp. 265-277.

61. D. Toledo, Harmonic maps from surfaces to certain Kähler manifolds, Math. Scand. 45 (1979), 13-26.

62. Non-existence of certains closed complex geodesics in the moduli space of curves, Pacific. J. Math. 129 (1987), no. 1, 187-192.

63. _ Representations of surface groups in complex hyperbolic space, J. Diff. Geom. 29 (1989), no. 1, 125-133.

64. V. Turaev, A cocycle for the symplectic first Chern class and the Maslov index, Funct. Anal. Appl. 18 (1984), 35-39.

65. W. T. van Est, Group cohomology and Lie algebra cohomology in Lie groups, I, II, Nederl. Akad. Wetensch. Proc. Series A. \{56\}=Indag. Math. 15 (1953), 484-504.

66. A. Wienhard, Bounded cohomology and geometry, Ph. D. thesis, Universität Bonn, 2004. 
67. J.A. Wolf, Fine structure of Hermitian symmetric spaces, Symmetric spaces (Short Courses, Washington Univ., St. Louis, Mo., 1969-1970), Dekker, New York, 1972, pp. 271-357. Pure and App. Math., Vol. 8.

68. E. Z. Xia, The moduli of flat $U(p, 1)$ structures on Riemann surfaces, Geom. Dedicata 97 (2003), 33-43, Special volume dedicated to the memory of Hanna Miriam Sandler.

69. R. J. Zimmer, Amenable ergodic group actions and an application to Poisson boundaries of random walks, J. Funct. Anal. 27 (1978), 350-372.

70. L Ergodic theory and semisimple groups, Birkhäuser, Boston, 1984.

Marc Burger

e-mail:burger@math.ethz.ch

FIM, ETH Zentrum, Rämistrasse 101

CH-8092 Zürich, Switzerland

\author{
Alessandra Iozzi \\ e-mail:Alessandra.Iozzi@unibas.ch \\ Institut für Mathematik \\ Universität Basel, Rheinsprung 21 \\ CH-4051 Basel, Switzerland \\ or \\ Départment de Mathématiques \\ Université de Strasbourg, 7, rue René Descartes \\ F-67084 Strasbourg Cedex, France
}

François Labourie

e-mail:labourie@math.u-psud.fr

Topologie et Dynamique, Bâtiment 425

Université de Paris Sud

F-91405 Orsay Cedex, France

Anna Wienhard

e-mail:Anna.Wienhard@unibas.ch

Institut für Mathematik

Universität Basel, Rheinsprung 21

CH-4051 Basel, Switzerland 